GCR $-96 / 07 / 02$

DTP-MSU/96-11

\title{
STATIONARY BPS SOLUTIONS TO DILATON-AXION GRAVITY
}

\author{
Gérard Clément*1 and Dmitri Gal'tsov ${ }^{\dagger 2}$ \\ 1 Laboratoire de Gravitation et Cosmologie Relativistes, \\ Université Pierre et Marie Curie, CNRS/URA769 \\ Tour 22-12, Boîte 142, 4 place Jussieu, 75252 Paris cedex 05, France \\ 2 Department of Theoretical Physics, Physics Faculty, \\ Moscow State University, 119899, Moscow, Russia
}

July 2, 1996

\begin{abstract}
Stationary four-dimensional BPS solutions to gravity coupled bosonic theories admitting a three-dimensional sigma-model representation on coset spaces are interpreted as null geodesics of the target manifold equipped with a certain number of harmonic maps. For asymptotically flat (or Taub-NUT) space-times such geodesics can be directly parametrized in terms of charges saturating the Bogomol'nyi-Gibbons-Hull bound, and classified according to the structure of related coset matrices. We investigate in detail the "dilatonaxion gravity" with one vector field, and show that in the space of BPS solutions an $S O(1,2) \times S O(2)$ classical symmetry is acting. Within the present formalism the most general multicenter (IWP/Taub-NUT dyon) solutions are derived in a simple way. We also discover a large new class of asymptotically flat solutions for which the dilaton and axion charges are constrained only by the BPS bound. The string metrics for these solutions are generically regular. Both the IWP class and the new class contain massless solutions.
\end{abstract}

PACS number(s): 97.60.Lf, 04.60.+n, 11.17.+y

*E-mail: gecl@ccr.jussieu.fr.

${ }^{\dagger}$ E-mail: galtsov@grg.phys.msu.su 


\section{Introduction}

A typical feature of many non-linear field theories is the existence of certain positivity bounds for the energy similar to the Bogomol'nyi-Prasad-Sommerfield ( BPS) bound [1] in the YangMills-Higgs models admitting monopoles. Classical solutions saturating these bounds (BPS solutions) possess topological charges and minimize the energy for given values of these charges. Consequently they are stable, and usually associated with solitons. Physically the BPS saturation condition means that forces between them are balanced, so generically the corresponding multisoliton solutions also exist. In supersymmetric embeddings solitons possess unbroken supersymmetries [2] which are manifest in the existence of Killing spinors constant at spatial infinity. The vanishing of the corresponding spinorial variations on soliton backgrounds gives rise to linear Bogomol'nyi type equations which facilitate the effective construction of these solitons. Topological charges enter as central charges into the supersymmetry algebra and hence the knowledge of the soliton spectrum is important for a proper understanding of the dynamics of the theory.

Similar phenomena are also well known in theories including gravity. The Einstein theory can be embedded in simple supergravity, the corresponding positivity argument providing a concise proof of the positive mass conjecture [3]. The importance of BPS bounds in extended supergravities was advocated by Gibbons 淊. In $N=2$ supergravity (containing in the bosonic sector the graviton and an Abelian vector field) the extreme Reissner-Nordström black hole saturates the BPS bound and has an unbroken supersymmetry, while the general non-singular solution of the corresponding spinorial equation is the Majumdar-Papapetrou multicenter solution to the Einstein-Maxwell (EM) equations [5]. Since quantum corrections to supersymmetric backgrounds are controllable, the BPS solutions are an important tool to investigate the underlying quantum theory non-perturbatively [6].

The $N=2$ theory, however, has anomalies, so the non-renormalization argument is not fully applicable. The situation is better in $N=4$ four-dimensional supergravity, containing in the bosonic sector the graviton, two scalar fields (dilaton and axion) and six $U(1)$ vectors. The BPS bound for static asymptotically flat solutions to this theory was discussed by Gibbons [7]. Truncated models of this kind containing one (dilaton-axion gravity), or two vector fields were extensively studied recently by Kallosh and collaborators [8] [9]. In [10] some stationary BPS solutions to dilaton-axion gravity were given and the BPS condition was generalized to include the NUT charge. Recently such solutions have been investigated in the context of heterotic string theory, which gives rise in four dimensions to a more general bosonic lagrangian including 28 vector fields, the dilaton and the axion and 132 scalar moduli fields forming a coset $S O(6,22) / S(O(6) \times O(22))$. The BPS bound in this theory was discussed in [11], some static spherically symmetric solutions saturating this bound were studied in [12]. Of particular interest are massless BPS states, which can substantially affect the low-energy dynamics 13].

In all listed theories there are internal symmetries relating configurations depending effectively on three space-time coordinates (e.g. static or stationary), which can be used to generate new explicit solutions. This was one of the main technical tools in most of the above papers. Another widely used approach was dimensional reduction ¿from ten-dimensional solutions known to be exact string backgrounds, such as stringy plane waves [10]. Both methods generically give physically interesting, but nevertheless restricted classes of solutions. A more general approach consists in the direct use of the first order equations for the supercovariantly constant spinors. Along these lines Tod was able to find all stationary BPS solutions to EM theory, dilaton gravity, and a large (although restricted) class of solutions to $N=4$ supergrav-

ity [14]. However, the complete enumeration of all stationary asymptotically flat solutions to dilaton-axion gravity (and larger theories including moduli fields) seems not to have been given so far. Here we make some progress in this direction using an alternative (purely bosonic) way 
to classify the BPS solutions.

The method utilizes the $\sigma$-model structure of the three-dimensional theories describing four-dimensional backgrounds admitting a non-null Killing vector. For pure gravity this is the well-known Ernst $S L(2, R) / S O(2) \sigma$-model [15], generalized to Maison's $S L(n-2, R) / S O(n-$ 2) $\sigma$-model [16] for $n$-dimensional vacuum Kaluza-Klein (KK) theory, while for the EM theory this is the Neugebauer and Kramer $S U(2,1) / S(U(2) \times U(1))$ model [17], [18]. More general classes of such models following from supergravities were studied by Breitenlohner, Maison and Gibbons [19]. The particular case of dilaton-axion gravity (with one vector field) was studied in [20], where the coset structure $S p(4, R) / U(2)$ of the corresponding target space was found. Typically, when the Killing vector, with respect to which a reduction to three dimensions is performed, is timelike, the resulting target space has an indefinite metric and contains null geodesic lines. I It is well known that geodesics of the target space equipped with some harmonic functions on a three-space generate a solution to the $\sigma$-model equations [17], [21]. It was observed in [22] that null geodesics of the target space of stationary fivedimensional KK theory may be used to generate multisoliton solutions similar to the IsraelWilson-Perjès solutions [23] of EM theory. The association of stationary multisoliton systems with null geodesics in target space is a general feature of $\sigma$-models, due to the fact that in the null case the three-space is conformally flat. This property can be related to the BPS force balance condition. Therefore, the classification of stationary BPS solutions may be traced to the investigation of the null geodesic structure of the three-dimensional $\sigma$-model target space.

We discuss the general features of this approach in Sect. 2, and then consider in detail the stationary dilaton-axion gravity model in Sect. 3. It is shown that isotropic geodesics in the space $S p(4, R) / U(2)$ fall into two classes depending on whether the the corresponding generating matrix is degenerate or not. The space of classical BPS solutions exhibits a symmetry under $S O(1,2) \times S O(2)$ transformations mapping one asymptotically Taub-NUT geodesic solution onto another (Sect. 4). These transformations include electric-magnetic duality, mass - NUT duality as well as two special boost transformations in the non-degenerate case. Solutions related to degenerate matrices are shown to reproduce all previously known BPS solutions including rotating dilaton-axion Taub-NUT dyon [25] and dilaton-axion IWP solutions [10], generalized to include all possible charges explicitly and to allow for arbitrary directions of rotation axes. Although the Einstein metrics for these extreme black hole solutions are singular, the corresponding string metrics are generically regular in the non-rotating case. Massless states with non-zero NUT-s are found. Non-degenerate matrices correspond to formally quasi-periodic solutions (Sect. 6). These solutions have dilaton and axion charges constrained only by the BPS condition. Their Einstein metrics present weak naked singularities, while the associated string metrics are again generically regular. Here we present these entirely new solutions in some detail, in view of their amazing simplicity. In Appendix A we describe a useful decomposition of the symplectic algebra, and find some intrinsic connection with $1+2$ Clifford algebras. In Appendix B the IWP solutions to the EM theory are rederived within the same approach.

\section{General formalism}

Consider a four-dimensional action

$$
S=\int\left(-\frac{R}{16 \pi}+L\left(A^{I}, \phi^{a}\right)\right) \sqrt{-g} d^{4} x
$$

\footnotetext{
${ }^{1}$ Note that the canonical procedure gives rise in the case of dilaton-axion gravity to the coset $S p(4, R) / U(1,1)$ [19], but the indefinite metric can also be presented in terms of symmetric matrices belonging to $S p(4, R) / U(2)$ which are somewhat simpler. In what follows we assume this representation of the non-compact target space of stationary dilaton-axion gravity.
} 
where $A^{I}$ denotes the set of $U(1)$ (linear) vector fields, and $\phi^{a}$ is the set of scalar fields typically including the dilaton, Peccei-Quinn axion and moduli. In extended supergravities/superstring effective theories scalar fields usually form coset spaces, while vector fields transform under a representation of the corresponding global symmetry group. When the theory is reduced to three dimensions, vectors can be traded for pairs of scalar fields, which can sometimes be incorporated into a larger coset space. The corresponding three-dimensional theory then turns out to be a gravity coupled sigma-model on a symmetric space. A general discussion and a list of different group combinations ensuring such a property were given by Julia [26] and Breitenlohner, Maison and Gibbons [19] and discussed later in a number of papers. Assuming a certain familiarity with this formalism, we just briefly recall here the basic formulas with an emphasis on the so-called geodesic solutions.

We will be interested in stationary solutions depending on three (space-like) coordinates, or, in other words, admitting an (asymptotically) timelike Killing vector field. Then the standard dimensional reduction ansatz

$$
g_{\mu \nu}=\left(\begin{array}{cc}
f & -f \omega_{i} \\
-f \omega_{i} & -f^{-1} h_{i j}+f \omega_{i} \omega_{j}
\end{array}\right)
$$

leads to the corresponding three-dimensional theory. In the stationary case, $U(1)$ vector fields may be expressed in terms of electric $v^{I}$ and magnetic $u^{I}$ potentials, while the rotation one-form $\omega=\omega_{i} d x^{i}$ can be dualized to give the twist potential $\chi$, the pair $(f, \chi)$ forming a coset space $S L(2, R) / S O(2)$ [17]. To make $u^{I}$ and $\chi$ dynamical variables one has to introduce Lagrange multipliers ensuring the Bianchi identities. The resulting action will be that of the gravity coupled three-dimensional $\sigma$-model

$$
S_{\sigma}=\frac{1}{2} \int\left(\mathcal{R}-G_{A B} \partial_{i} X^{A} \partial_{j} X^{B} h^{i j}\right) \sqrt{h} d^{3} x,
$$

where $\mathcal{R}$ is the Ricci scalar built out of $h_{i j}$, and the set of scalars $X^{A}=\left(f, \chi, v^{I}, u^{I}, \phi^{a}\right)$, combined into the potential space endowed with the metric $G_{A B}(X)$, acts as a source of threedimensional Euclidean gravity. The equations of motion for the $X^{A}$

$$
\frac{1}{\sqrt{h}} \partial_{i}\left(\sqrt{h} h^{i j} G_{A B} \partial_{j} X^{B}\right)=0
$$

define a harmonic map from the three-space $\left\{x^{i}\right\}$ to the potential space $\left\{X^{A}\right\}$ (target space).

Obviously, the equations of motion (2.4) are preserved by the isometry group $G$ of the target manifold. Therefore this group is a symmetry group of the space of solutions, using which new solutions may be generated. In the class of theories under consideration this group is large enough to ensure the symmetric space property of the target space, in which case the latter may be regarded as a coset space $G / H$, with $H$ a subgroup of $G$. This implies the existence of a matrix $M$ (an appropriate coset representative) in terms of which the target space metric is given by

$$
d l^{2}=G_{A B} d X^{A} d X^{B}=-\frac{1}{4} \operatorname{Tr}\left(d M d M^{-1}\right) .
$$

Consequently, the three-dimensional action will read

$$
S_{\sigma}=\frac{1}{2} \int\left\{\mathcal{R}+\frac{1}{4} \operatorname{Tr}\left(\nabla M \nabla M^{-1}\right)\right\} \sqrt{h} d^{3} x,
$$

where $\boldsymbol{\nabla}$ stands for the three-dimensional covariant derivative, and a scalar product with respect to the metric $h_{i j}$ is understood. The corresponding equations of motion take the form of the conservation equation for the matrix current

$$
\nabla\left(M^{-1} \nabla M\right)=0
$$


while the three-dimensional Einstein equations read

$$
\mathcal{R}_{i j}=-\frac{1}{4} \operatorname{Tr}\left(\nabla_{i} M \nabla_{j} M^{-1}\right) .
$$

As was noticed by Neugebauer and Kramer [17], when one makes the special assumption that all target space coordinates $X^{A}$ depend on $x^{i}$ through only one scalar potential, i.e. $X^{A}=X^{A}\left[\sigma\left(x^{i}\right)\right]$, it follows from the equation of motion (2.4) that this potential can be chosen to be harmonic,

$$
\Delta \sigma=0, \quad \Delta=\nabla^{2},
$$

Eq. (2.4) reducing then to the geodesic equation on the target space

$$
\frac{d^{2} X^{A}}{d \sigma^{2}}+\Gamma_{B C}^{A} \frac{d X^{B}}{d \sigma} \frac{d X^{C}}{d \sigma}=0
$$

Rewriting this in matrix terms,

$$
\frac{d}{d \sigma}\left(M^{-1} \frac{d M}{d \sigma}\right)=0
$$

one may express the solution to the geodesic equation in the exponential form

$$
M=A \mathrm{e}^{B \sigma},
$$

where $A \in G / H$ and $B \in \mathcal{L} i e(G)$ are constant matrices such that $M \in G / H$. Usually we are interested in asymptotically flat (Taub-NUT) solutions, and it is assumed that $\sigma(\infty)=0$. Then the asymptotic value of $M$ is $A$, some fixed quantity depending on the choice of the coset representative. Asymptotically flat solutions of this kind are thus target space geodesics passing through the point $A$.

The three-dimensional Einstein equations (2.8) read, in the case of geodesic solutions,

$$
\mathcal{R}_{i j}=\frac{1}{4} \operatorname{Tr}\left(B^{2}\right) \nabla_{i} \sigma \nabla_{j} \sigma
$$

From this expression it is clear that in the particular case

$$
\operatorname{Tr}\left(B^{2}\right)=0
$$

the three-space is Ricci-flat. In three dimensions the Riemann tensor is then also zero, and consequently the three-space is flat. From the Eq. (2.5) one can see that the condition 2.14 corresponds to null geodesics [22] of the target space

$$
d l^{2}=\frac{1}{4} \operatorname{Tr}\left(B^{2}\right) d \sigma^{2}=0 .
$$

For different particular theories it was observed that null geodesics describe extremal black holes satisfying a Bogomol'nyi type condition on the relevant charges. The flat nature of the three space supports the anticipation that the attractive/repulsive forces associated with different charges are mutually balanced. In cases when the bosonic action can be embedded into some supergravity, it was argued that this condition corresponds to the existence of Killing spinors [6] [14] ensuring the unbroken supersymmetries of bosonic solutions (usually called BPS solutions). Although no general proof is known so far that any BPS solution can be presented as a null geodesic passing through $A$, at least the inverse statement seems to be rather plausible [19].

Whereas the group $G$ exhibits a symmetry of the full solution space, only a restricted set of $G$-transformations preserves the class of asymptotically flat geodesic solutions. To find the 
corresponding subgroup (in particular, we are interested in symmetries of the BPS subspace), consider a similarity transformation for the matrix $B$

$$
B \rightarrow B_{U}=U B U^{-1}, \quad U \in G,
$$

and construct the corresponding

$$
M_{U}=A \mathrm{e}^{B_{U} \sigma} .
$$

Since $A$ is not transformed, $M_{U}$ is not necessarily an element of $G / H$. However for some $U$ it may occur that $M_{U} \in G / H$ indeed, and thus is a solution. Such $U$ will be the symmetry transformation mapping one geodesic solution onto another. The condition $\operatorname{Tr}\left(B_{U}^{2}\right)=0$ will be satisfied automatically, if (2.14) holds. Therefore we deal with symmetries of the BPS space as well.

The various null geodesic solutions fall into equivalence classes, corresponding to the inequivalent types of matrices $B$ satisfying (2.14). There is only one matrix type in the EM case, leading to the IWP class (see Appendix B). Three distinct matrix types are associated with null geodesics of the five-dimensional KK theory [22]. The first type yields regular quasi-periodic solutions, the regular solutions of the second type are extreme black holes, while the third class contains the KK magnetic monopoles and multipoles [24]. As we shall see in the next section, only two matrix types occur in dilaton-axion gravity with one vector field.

The construction (2.12) may be generalized [22] to the case of several harmonic functions $\sigma_{a}$

$$
\Delta \sigma_{a}=0
$$

by showing that

$$
M=A \exp \left(\sum_{a} B_{a} \sigma_{a}\right)
$$

solves the field equations (2.7) provided that the commutators $\left[B_{a}, B_{b}\right]$ commute with the $B_{c}$ :

$$
\left[\left[B_{a}, B_{b}\right], B_{c}\right]=0 .
$$

To prove this, we first rewrite (2.19) as

$$
M=A \exp \left(-\frac{1}{2} \sum_{c>b} \sum_{b}\left[B_{b}, B_{c}\right] \sigma_{b} \sigma_{c}\right) \prod_{a} \mathrm{e}^{B_{a} \sigma_{a}}
$$

where the first exponential may be treated as a c-number function. We then obtain for the matrix current

$$
M^{-1} \nabla M=\sum_{a} B_{a} \nabla \sigma_{a}-\frac{1}{2} \sum_{c>b} \sum_{b}\left[B_{b}, B_{c}\right]\left(\sigma_{b} \nabla \sigma_{c}-\sigma_{c} \nabla \sigma_{b}\right),
$$

which is conserved if the $\sigma_{a}$ are harmonic. The three-dimensional Einstein equations (2.8) generalize to

$$
R_{i j}=\frac{1}{4} \sum_{a} \sum_{b} \operatorname{Tr}\left(B_{a} B_{b}\right) \nabla_{i} \sigma_{a} \nabla_{j} \sigma_{b},
$$

so that the three-space is Ricci flat if the matrices $B_{a}$ satisfy

$$
\operatorname{Tr}\left(B_{a} B_{b}\right)=0 .
$$

So the number of independent harmonic functions on which may depend a BPS solution of the form (2.19) is limited by the number of independent mutually orthogonal null vectors of the target space. In the class of theories we are dealing with, the target space is a locally Minkowskian space $M_{p, q}$, where $p$ counts the positive eigenvalues coming from the two gravitational potentials $f, \chi$ and the effective scalar fields, and $q$ is the number of negative eigenvalues coming from electric and magnetic potentials. Then the number of independent null vectors is $\inf (p, q)$. The actual number of possible independent potentials, however, may be less because of the extra condition $(2.20)$. 


\section{Dilaton-axion gravity}

In the following we turn to a specific theory which may be regarded as a (truncated) bosonic sector of the four-dimensional heterotic string effective action, or that of the $N=4, D=4$ supergravity with only one non-zero vector field. The model describes the gravity-coupled system of two scalar fields (dilaton $\phi$ and (pseudoscalar) axion $\kappa$ ), and one Abelian vector field $A_{\mu}$ :

$$
S=\frac{1}{16 \pi} \int\left\{-R+2 \partial_{\mu} \phi \partial^{\mu} \phi+\frac{1}{2} e^{4 \phi} \partial_{\mu} \kappa \partial^{\mu} \kappa-e^{-2 \phi} F_{\mu \nu} F^{\mu \nu}-\kappa F_{\mu \nu} \tilde{F}^{\mu \nu}\right\} \sqrt{-g} d^{4} x,
$$

where $\tilde{F}^{\mu \nu}=\frac{1}{2} E^{\mu \nu \lambda \tau} F_{\lambda \tau}, F=d A$.

Assuming stationarity, one can perform reduction to three dimensions using the metric ansatz (2.2). The vector field can be parametrized by 2 real functions: an electric potential $v$,

$$
F_{i 0}=\partial_{i} v / \sqrt{2}
$$

and a magnetic potential $u$,

$$
e^{-2 \phi} F^{i j}+\kappa \tilde{F}^{i j}=f \epsilon^{i j k} \partial_{k} u / \sqrt{2 h} .
$$

The corresponding spatial vector potential $A_{i}$ may be recovered from $u$ and $v$ by

$$
\sqrt{2} d A=e^{2 \phi} f^{-1} *(d u-\kappa d v)-d v \wedge \omega .
$$

To satisfy the mixed components of the four-dimensional Einstein equations one has to express $\omega_{k}$ through the twist potential $\chi$ as follows [25]

$$
\tau_{i}=\partial_{i} \chi+v \partial_{i} u-u \partial_{i} v, \quad \tau^{i}=-f^{2} \epsilon^{i j k} \partial_{j} \omega_{k} / \sqrt{h} .
$$

Here and below 3-indices are raised and lowered using the three-space (euclidean signature) metric $h_{i j}$ and its inverse $h^{i j}$.

The resulting three-dimensional gravity coupled $\sigma$-model has a six-dimensional target space, $X^{A}=(f, \chi, v, u, \phi, \kappa), A=1, \ldots, 6$ endowed with the metric

$$
d l^{2}=\frac{1}{2 f^{2}}\left\{d f^{2}+(d \chi+v d u-u d v)^{2}\right\}-\frac{1}{f}\left\{e^{-2 \phi} d v^{2}+e^{2 \phi}(d u-\kappa d v)^{2}\right\}+2 d \phi^{2}+\frac{1}{2} e^{4 \phi} d \kappa^{2}
$$

A representation similar to (3.6) has been found for the stationary EM system by Neugebauer and Kramer [17], their formula can be recovered by setting $\phi=\kappa=0$. As was shown by Mazur [18], the EM target space is isomorphic to the symmetric space $S U(2,1) / S(U(2) \times U(1))$. When the dilaton and axion fields are introduced one obtains instead the six-dimensional symmetric space $S p(4, R) / U(2)$ 20]. A matrix representative of this coset can be chosen to be the symmetric symplectic matrix [27]

$$
M=\left(\begin{array}{cr}
P^{-1} & P^{-1} Q \\
Q P^{-1} & P+Q P^{-1} Q
\end{array}\right),
$$

where $P$ and $Q$ are the real symmetric $2 \times 2$ matrices

$$
P=-\mathrm{e}^{-2 \phi}\left(\begin{array}{cc}
v^{2}-f \mathrm{e}^{2 \phi} & v \\
v & 1
\end{array}\right), \quad P^{-1}=f^{-1}\left(\begin{array}{cc}
1 & -v \\
-v & v^{2}-f \mathrm{e}^{2 \phi}
\end{array}\right), \quad Q=\left(\begin{array}{cc}
v w-\chi & w \\
w & -\kappa
\end{array}\right),
$$

with $w=u-\kappa v$. 
A solution $M$ depending on only one potential is a geodesic

$$
M=A \mathrm{e}^{B \sigma}
$$

passibg through a given point $A$. If we choose as usual the harmonic potential to vanish at spatial infinity, then $A=M(\infty)$. For the asymptotically flat and/or asymptotically TaubNUT configurations we are interested in, the "potentials" $X^{A}$ should be normalized at spatial infinity by

$$
f(\infty)=1, \quad \chi(\infty)=v(\infty)=u(\infty)=\phi(\infty)=\kappa(\infty)=0,
$$

leading to

$$
A=\left(\begin{array}{cc}
\sigma_{3} & 0 \\
0 & \sigma_{3}
\end{array}\right)
$$

(here and below $I$ is the $2 \times 2$ unit matrix, $\sigma_{1}=\sigma_{x}, \sigma_{2}=i \sigma_{y}, \sigma_{3}=\sigma_{z}$, and $\sigma_{x}, \sigma_{y}, \sigma_{z}$ are the standard Pauli matrices with $\sigma_{z}$ diagonal). Now we state the conditions on the matrix $B$ such that $M \in S p(4, R) / U(2)$. The symmetry of $M$ leads to the pseudosymmetry condition

$$
B^{T} A=A B .
$$

$M$ is also symplectic, $M^{T} J M=J$, with

$$
J=\left(\begin{array}{cc}
0 & I \\
-I & 0
\end{array}\right)
$$

On account of (3.12) and of the symplecticity of $A$, this leads to the condition

$$
B K+K B=0,
$$

with

$$
K=\left(\begin{array}{cc}
0 & \sigma_{3} \\
-\sigma_{3} & 0
\end{array}\right)
$$

Finally, $M$ is unimodular, leading to

$$
\operatorname{Tr} B=0 .
$$

As shown in Appendix A, the exponential $\mathrm{e}^{B \sigma}$ belongs to the coset $S p(4, R) /(S O(2) \times$ $S O(1,2))$, where the $S O(2)$ component is generated by $K$, while the remaining non-compact group is generated by the $\Sigma_{a}$,

$$
\Sigma_{a}=\left\{\left(\begin{array}{cc}
0 & I \\
-I & 0
\end{array}\right), \quad\left(\begin{array}{cc}
0 & -\sigma_{1} \\
-\sigma_{1} & 0
\end{array}\right), \quad\left(\begin{array}{cc}
\sigma_{1} & 0 \\
0 & -\sigma_{1}
\end{array}\right)\right\}
$$

(note that $\Sigma_{0} \equiv J$ ), with the commutation relations

$$
\left[\Sigma_{a}, \Sigma_{b}\right]=2 \varepsilon_{a b}{ }^{c} \Sigma_{c}, \quad\left[K, \Sigma_{a}\right]=0 .
$$

Here and below we use $S O(1,2)$ indices $a, b, c=0,1,2$ which are lowered/raised using the $1+2$ Minkowski metric $\eta_{a b}=\operatorname{diag}(1,-1,-1)$ and its inverse $\eta^{a b}=\eta_{a b}$.

The six remaining elements of $s p(4, R)$ can be cast into two $s o(1,2)$ (co)vectors

$$
\Gamma_{a}^{1}=\left\{\left(\begin{array}{cc}
0 & \sigma_{1} \\
-\sigma_{1} & 0
\end{array}\right), \quad\left(\begin{array}{cc}
0 & -I \\
-I & 0
\end{array}\right), \quad\left(\begin{array}{cc}
I & 0 \\
0 & -I
\end{array}\right)\right\}
$$

and

$$
\Gamma_{a}^{2}=\left\{\left(\begin{array}{cc}
\sigma_{2} & 0 \\
0 & \sigma_{2}
\end{array}\right), \quad\left(\begin{array}{cc}
\sigma_{3} & 0 \\
0 & -\sigma_{3}
\end{array}\right), \quad\left(\begin{array}{cc}
0 & \sigma_{3} \\
\sigma_{3} & 0
\end{array}\right)\right\},
$$

satisfying the commutation relations (A.14), A.15) with $K$ and $\Sigma_{a}$. 
Therefore, the most general $B$ can be parametrized by two $S O(1,2)$ vectors $\boldsymbol{\alpha}, \boldsymbol{\beta}$ as follows

$$
B=\alpha^{a} \Gamma_{a}^{1}+\beta^{a} \Gamma_{a}^{2} \equiv \boldsymbol{\alpha} \cdot \boldsymbol{\Gamma}^{1}+\boldsymbol{\beta} \cdot \boldsymbol{\Gamma}^{2} .
$$

To perform exponentiation one needs an anticommutator

$$
\left\{\Gamma_{a}^{1}, \Gamma_{b}^{2}\right\}=-2 \varepsilon_{a b}^{c} \eta^{c d} \Gamma_{d}^{0}
$$

which lies outside the Lie algebra $s p(4, R)$ :

$$
\Gamma_{a}^{0}=\left\{\left(\begin{array}{cc}
\sigma_{3} & 0 \\
0 & \sigma_{3}
\end{array}\right), \quad\left(\begin{array}{cc}
\sigma_{2} & 0 \\
0 & -\sigma_{2}
\end{array}\right), \quad\left(\begin{array}{cc}
0 & \sigma_{2} \\
\sigma_{2} & 0
\end{array}\right)\right\} .
$$

Remarkably, this third triplet of matrices makes the full system $S O(1,2)$ covariant on the upper index as well, as discussed in Appendix A. The anticommutator

$$
\left\{\Gamma_{a}^{c}, \Gamma_{b}^{d}\right\}=2\left(\eta^{c d} \eta_{a b} I-\varepsilon^{c d}{ }_{f} \varepsilon_{a b}{ }^{e} \Gamma_{e}^{f}{ }_{e}\right)
$$

is valid for all $c, d$. This last relation shows that the three sets of gamma's form $1+2$ Clifford algebras.

Using these formulas one immediately obtains for the square of (3.21)

$$
B^{2}=-\left(\boldsymbol{\alpha}^{2}+\boldsymbol{\beta}^{2}\right) I-2(\boldsymbol{\alpha} \wedge \boldsymbol{\beta}) \cdot \boldsymbol{\Gamma}^{0}
$$

so that the null geodesic condition (2.14) reads

$$
\operatorname{Tr}\left(B^{2}\right)=-4\left(\boldsymbol{\alpha}^{2}+\boldsymbol{\beta}^{2}\right)=0 .
$$

Assuming this condition to hold, we obtain successively

$$
B^{3}=-2\left(\boldsymbol{\alpha}^{\prime} \cdot \boldsymbol{\Gamma}^{1}+\boldsymbol{\beta}^{\prime} \cdot \boldsymbol{\Gamma}^{2}\right)
$$

where

$$
\boldsymbol{\alpha}^{\prime}=\boldsymbol{\beta} \wedge(\boldsymbol{\alpha} \wedge \boldsymbol{\beta}), \quad \boldsymbol{\beta}^{\prime}=\boldsymbol{\alpha} \wedge(\boldsymbol{\beta} \wedge \boldsymbol{\alpha})
$$

and

$$
B^{4}=4(\boldsymbol{\alpha} \wedge \boldsymbol{\beta})^{2} I
$$

The fact that the matrix $B^{4}$ is proportional to unity could also have been derived from the matrix identity

$$
\mathcal{P}(B)=0
$$

where

$$
\mathcal{P}(\lambda)=\operatorname{det}(B-\lambda I)=\prod_{i=1}^{4}\left(\lambda-\lambda_{i}\right)
$$

is the characteristic polynomial. Because the matrix $B$ is constrained by equations $(3.16)$ and (3.26), $\operatorname{Tr} B=0$ and $\operatorname{Tr}\left(B^{2}\right)=0$, leading to $\operatorname{Tr}\left(B^{3}\right)=0$ as well from eq. (3.27), only zero and fourth powers of $\lambda$ enter this characteristic polynomial. Consequently, the identity (3.30) reads

$$
B^{4}+\operatorname{det} B=0 \text {. }
$$

Now we are in a position to classify the possible types of matrices $B$ satisfying the null geodesic condition (3.26). It follows from Eqs. (3.29), (3.26) and (3.32) that there are two essentially different types of $B$ matrices: 
i) Type 1: degenerate $B, \operatorname{det} B=0$.

This is the case whether 1a: $\boldsymbol{\alpha}$ and $\boldsymbol{\beta}$ are collinear,

$$
\boldsymbol{\beta}=c \boldsymbol{\alpha}, \quad 0<c<\infty, \quad \text { with } \quad \boldsymbol{\alpha}^{2}=0
$$

¿from Eq. 3.26), or $1 \mathrm{~b}: \boldsymbol{\alpha}=0, \boldsymbol{\beta}^{2}=0$ or vice versa (Eq. 3.33) goes over to this in the limit $c \rightarrow \infty$ or $c \rightarrow 0$ ). For the type $1, B^{2}=0$ and hence the expansion of the exponential $\exp (B \sigma)$ contains only a linear term.

ii) Type 2: non-degenerate $B, \operatorname{det} B \neq 0$.

type $2 \mathrm{a}: \boldsymbol{\alpha}$ and $\boldsymbol{\beta}$ are non-collinear, but both are null, i.e. $\boldsymbol{\alpha}^{2}=0, \boldsymbol{\beta}^{2}=0$;

type $2 \mathrm{~b}$ : neither of $\boldsymbol{\alpha}$ and $\boldsymbol{\beta}$ is null, that is either

$$
\boldsymbol{\alpha}^{2}=-\boldsymbol{\beta}^{2}>0
$$

or

$$
\boldsymbol{\beta}^{2}=-\boldsymbol{\alpha}^{2}>0
$$

As we will see later, there is an internal symmetry of the space of geodesic solutions mixing subclasses $a$ and $b$ inside each type, but preserving the distinction between types 1 and 2 .

Now we look for BPS solutions depending on two harmonic functions $\sigma, \tau$

$$
M=A \mathrm{e}^{B \sigma+C \tau},
$$

with $C$ satisfying the same conditions as $B$, and thus admitting an expansion

$$
C=\gamma \cdot \Gamma^{1}+\delta \cdot \Gamma^{2}
$$

and, in addition?],

$$
[B, C]=0, \quad \operatorname{Tr}(B C)=0 .
$$

These last equations impose the following conditions on the $S O(1,2)$ vectors $\boldsymbol{\gamma}, \boldsymbol{\delta}$ :

$$
\begin{aligned}
\boldsymbol{\alpha} \wedge \boldsymbol{\gamma}+\boldsymbol{\beta} \wedge \boldsymbol{\delta} & =0 \\
\boldsymbol{\alpha} \cdot \boldsymbol{\delta}-\boldsymbol{\beta} \cdot \boldsymbol{\gamma} & =0 \\
\boldsymbol{\alpha} \cdot \boldsymbol{\gamma}+\boldsymbol{\beta} \cdot \boldsymbol{\delta} & =0
\end{aligned}
$$

These conditions lead to very strong restrictions on possible $\boldsymbol{\gamma}, \boldsymbol{\delta}$ once $\boldsymbol{\alpha}, \boldsymbol{\beta}$ are given. Namely, for $B$ of type 1, the matrix $C$ should be also of type 1, and $\boldsymbol{\gamma}, \boldsymbol{\delta}$ should be collinear to $\boldsymbol{\alpha}, \boldsymbol{\beta}$; it then follows that $B C=C B=0$. Redefining $\sigma, \tau$ through suitable linear combinations one can then always choose

$$
B=(0, \boldsymbol{\beta}), \quad C=(\boldsymbol{\beta}, 0), \quad \text { with } \quad \boldsymbol{\beta}^{2}=0 .
$$

Since for the type 1 the exponents in (3.36) contain only linear terms it is clear that the most general solution can be presented in the equivalent form

$$
M=A\left\{1+\boldsymbol{\beta} \cdot\left(\boldsymbol{\Gamma}^{1} \tau+\boldsymbol{\Gamma}^{2} \sigma\right)\right\} .
$$

If $B$ is of type 2 , then the only solution is $C \propto B$ (i.e. there is no extreme solution really depending on two potentials). Since the target space (3.6) is locally $M_{4,2}$, it is also clear that for the present system no BPS solutions depending on more than two potentials are possible.

\footnotetext{
${ }^{2}$ The study of the Lie algebra $\operatorname{sp}(4, \mathrm{R})$ shows that the more general condition $(2.19)$ on the commutator $[B, C]$ reduces to $[B, C]=0$.
} 


\section{Physical charges and $S O(1,2) \times S O(2)$ symmetry of BPS space}

From the above analysis it follows that any stationary asymptotically flat BPS solution to dilaton-axion gravity can be parametrized by six real numbers subject to the null-geodesic condition (3.26). These numbers can be conveniently arranged into two $S O(1,2)$ vectors $\boldsymbol{\alpha}, \boldsymbol{\beta}$. Here we want to relate them to physical charges which can be introduced via the standard asymptotic expansions of the relevant fields in the asymptotically Taub-NUT space-time (including the usual asymptotically flat one as a particular case). Here we introduce the charges in accordance with the paper [25] as follows (recall the gauge conditions (3.10) )

$$
\begin{aligned}
& f \sim 1-\frac{2 M}{r}, \\
& \chi \sim-\frac{2 N}{r}, \\
& v \sim \frac{\sqrt{2} Q}{r}, \\
& u \sim \frac{\sqrt{2} P}{r}, \\
& \phi \sim \frac{D}{r}, \\
& \kappa \sim \frac{2 A}{r},
\end{aligned}
$$

It is also convenient to use a complex mass combining the Schwarzschild mass $M$ and the NUT charge $N$ :

$$
m=M+i N,
$$

a complex axidilaton charge combining the dilaton $D$ and axion $A$ charges

$$
d=D+i A
$$

and a complex electromagnetic charge

$$
q=Q+i P
$$

joining the electric $Q$ and magnetic $P$ charges.

To make the identification of the components of the vectors $\boldsymbol{\alpha}, \boldsymbol{\beta}$ with physical charges, we consider a solution (2.12) depending on a single monopole potential $\sigma$, with the matrix $B$ of the generic form (3.21). We choose the vectors $\boldsymbol{\alpha}, \boldsymbol{\beta}$ to have the dimension of a length (i.e. the

dimension of the charges in (4.1)). The dimension of the harmonic function $\sigma$ will then be an inverse length, and we choose the normalization

$$
\sigma \sim \frac{1}{r}
$$

Substituting the expansions (4.1) into the matrix $M$ in the form (3.7), and comparing with the corresponding linearization of (3.9), one finds the following correspondence

$$
\begin{aligned}
& \boldsymbol{\alpha}=(\sqrt{2} P, A-N, M+D), \\
& \boldsymbol{\beta}=(-\sqrt{2} Q, M-D, N+A) .
\end{aligned}
$$

The null geodesic (BPS) condition (3.26) $\boldsymbol{\alpha}^{2}+\boldsymbol{\beta}^{2}=0$ then assumes its standard form

$$
Q^{2}+P^{2}=M^{2}+N^{2}+D^{2}+A^{2},
$$


or, in complex notation,

$$
|q|^{2}=|m|^{2}+|d|^{2} .
$$

For type 1 and type 2 a some additional constraints are imposed on $\boldsymbol{\alpha}, \boldsymbol{\beta}$. Consider first the degenerate type 1 . The collinearity condition (without assuming the BPS constraint (4.7)) gives

$$
\begin{gathered}
M^{2}+N^{2}=D^{2}+A^{2}, \\
Q(N-A)=P(M-D), \\
P(N+A)=-Q(M+D)
\end{gathered}
$$

(only two of these equations are independent). Solving these equations with respect to $D, A$ one gets

$$
\begin{aligned}
& D=\frac{M\left(P^{2}-Q^{2}\right)-2 N Q P}{Q^{2}+P^{2}}, \\
& A=\frac{N\left(Q^{2}-P^{2}\right)-2 M Q P}{Q^{2}+P^{2}},
\end{aligned}
$$

or, in complex form

$$
d=-\frac{\bar{m} q}{\bar{q}} .
$$

Now, if the BPS condition (4.7) is imposed, one gets from (4.9)

$$
Q^{2}+P^{2}=2\left(M^{2}+N^{2}\right),
$$

or $\bar{q} q=2 \bar{m} m$, so that the relation (4.13) takes the "holomorphic" form

$$
d=-\frac{q^{2}}{2 m}
$$

This relation was found for a rotating Taub-NUT dyon in dilaton-axion gravity in [25]. Particular cases of these dyon configurations are the purely electric type $P=0(\boldsymbol{\alpha}=0)$, for which the extremal states have dilaton and axion charges

$$
D=-M, A=N,
$$

and the purely magnetic type $Q=0(\boldsymbol{\beta}=0)$, for which one has

$$
D=M, A=-N \text {. }
$$

Let us now consider the possibility of solutions with zero Schwarzschild mass. Within the type 1 such non-trivial configurations may exist if the NUT parameter is non-zero. In the generic dyon case (type 1a) the dilaton and axion charges are

$$
D=-\frac{Q P}{N}, A=\frac{Q^{2}-P^{2}}{2 N}
$$

and the BPS condition reads $Q^{2}+P^{2}=2 N^{2}$ (magnetic solutions of this kind were given in [31]). In non-dyon cases

$$
D=0, \quad A= \pm N,
$$

where the upper sign coresponds to electric and lower to magnetic solutions. 
Now we discuss the relations between charges for solutions corresponding to non-degenerate $B$. In the null case $2 \mathrm{a}$ one has in addition to the BPS condition (4.7) a single constraint

$$
M D-N A=\frac{P^{2}-Q^{2}}{2} .
$$

Contrary to type 1 , in the case 2 a generically

$$
M^{2}+N^{2} \neq A^{2}+D^{2}
$$

(if the equality holds we come back to the case 1a). Solving (4.7) and (4.20) we obtain for the dilaton and axion charges in the case $2 \mathrm{a}$

$$
\begin{aligned}
D & =\frac{M\left(P^{2}-Q^{2}\right)-2 N Q P \varrho}{2\left(M^{2}+N^{2}\right)}, \\
A & =\frac{N\left(Q^{2}-P^{2}\right)-2 M Q P \varrho}{2\left(M^{2}+N^{2}\right)}
\end{aligned}
$$

where

$$
\varrho^{2}=1-\frac{\left[Q^{2}+P^{2}-2\left(M^{2}+N^{2}\right)\right]^{2}}{4 Q^{2} P^{2}} .
$$

Comparing this with the "standard" relations (4.12) one can see their difference unless $\rho=1$ in which case we come back to the type 1 . Finally, in the non-null non-degenerate type $2 \mathrm{~b}$ case the physical charges are constrained only by the BPS condition (4.7).

Now let us discuss the symmetry transformations acting inside the class of stationary asymptotically Taub-NUT geodesic solutions to dilaton-axion gravity. As was explained in Sect. 2, we look for similarity transformations for the matrix $B$ which preserve the pseudosymmetry condition (3.12). It is shown in the Appendix A that if $M \in S p(4, R) / U(2)$ and $A$ is given by (3.11), then $A M=\mathrm{e}^{B \sigma} \in S p(4, R) /(S O(1,2) \times S O(2))$. Therefore the subgroup $H^{\prime}=S O(1,2) \times S O(2)$ is the desired symmetry group. In algebraic terms, to preserve the condition (3.12), the generator $X \in \operatorname{sp}(4, R)$ of the similarity transformation (2.16) should satisfy

$$
X^{T} A=-A X
$$

In view of the decomposition of the $\operatorname{sp}(4, R)$ algebra described in Appendix A, it is clear that $X \in \mathcal{H}^{\prime}$ (cf. (A.11)). In fact this subgroup of the symplectic group has a larger meaning as that preserving asymptotic flatness (so it is also relevant to non-null geodesic solutions). It is worth giving a more detailed description of this symmetry with an emphasis on its action on BPS solutions.

The group $H^{\prime}$ consists of two components. The first one $(S O(2))$ is generated by $K$, i.e. in (2.16) $U=\mathrm{e}^{K \epsilon / 2}$. This transformation mixes $\boldsymbol{\alpha}$ and $\boldsymbol{\beta}$ :

$$
\begin{aligned}
& \boldsymbol{\alpha} \rightarrow \boldsymbol{\alpha} \cos \epsilon+\boldsymbol{\beta} \sin \epsilon, \\
& \boldsymbol{\beta} \rightarrow \boldsymbol{\beta} \cos \epsilon-\boldsymbol{\alpha} \sin \epsilon
\end{aligned}
$$

In terms of charges this is an electric-magnetic duality rotation, i.e. the transformed quantities are

$$
q^{\prime}=q \mathrm{e}^{-i \epsilon}, \quad m^{\prime}=m \mathrm{e}^{-i \epsilon}, \quad d^{\prime}=d \mathrm{e}^{-i \epsilon} .
$$

It is worth noting that when $Q$ and $P$ get mixed by this transformation, at the same time the mass and the NUT charge are also mixed, i.e. one is not allowed to make a dyon from a purely electric (purely magnetic) asymptotically flat (NUT-less) configuration without introducing a NUT charge. Clearly, the axidilaton charge follows the transformation rule implied by (4.15). 
Comparing this with the general case of the action of the symplectic group presented as the set of scale, gauge, $S$-duality, Harrison and Ehlers transformations [27], one can observe that the $K$-transformation is a certain combination of Ehlers, gravitational gauge and two $S$-duality transformations.

Now consider the $S O(1,2)$ part of $H^{\prime}$. It is generated by the $\Sigma_{a}$ according to (3.18). In view of (A.15), these are the transformations acting independently (and similarly) on $\boldsymbol{\alpha}$ and $\boldsymbol{\beta}$. Pure 'spatial' rotations are given by $U=\mathrm{e}^{\Sigma_{0} \theta / 2}$ :

$$
\begin{gathered}
\alpha^{0} \rightarrow \alpha^{0}, \\
\alpha^{1} \rightarrow \alpha^{1} \cos \theta+\alpha^{2} \sin \theta, \\
\alpha^{2} \rightarrow \alpha^{2} \cos \theta-\alpha^{1} \sin \theta
\end{gathered}
$$

(similarly for $\boldsymbol{\beta})$. In terms of charges we find

$$
\begin{aligned}
q^{\prime} & =q, \\
m^{\prime} & =m \mathrm{e}^{-i \theta}, \\
d^{\prime} & =d \mathrm{e}^{i \theta} .
\end{aligned}
$$

This transformation can be used to compensate the mass - NUT charge mixing when the $K-$ transformation is applied, if strict asymptotic flatness is desired to be preserved. On the other hand, this transformation may be used to generate a NUT charge for purely electric and purely magnetic configurations. It is also a (different) combination of Ehlers, gravitational gauge and two of $S$-duality transformations.

The two other $S O(1,2)$ transformations are boost-like, they have different actions on null and non-null $\boldsymbol{\alpha}$ and $\boldsymbol{\beta}$. Consider first the non-null case. The boost in the plane $0-1$ is generated by $U=e^{\Sigma_{2} \xi / 2}$ :

$$
\begin{gathered}
\alpha^{0} \rightarrow \alpha^{0} \cosh \xi-\alpha^{1} \sinh \xi, \\
\alpha^{1} \rightarrow \alpha^{1} \cosh \xi-\alpha^{0} \sinh \xi, \\
\alpha^{2} \rightarrow \alpha^{2} .
\end{gathered}
$$

Together with the corresponding transformation of $\boldsymbol{\beta}$ this results in

$$
\begin{aligned}
q^{\prime} & =q \cosh \xi+\frac{m-d}{\sqrt{2}} \sinh \xi \\
m^{\prime}+d^{\prime} & =m+d, \\
m^{\prime}-d^{\prime} & =(m-d) \cosh \xi+\sqrt{2} q \sinh \xi
\end{aligned}
$$

This is a combination of electric gauge and Harrison transformations which change all six charges in a non-trivial way. Similarly, the $0-2$ boost $U=e^{\Sigma_{1} \eta / 2}$ gives

$$
\begin{aligned}
q^{\prime} & =q \cosh \eta+i \frac{m+d}{\sqrt{2}} \sinh \eta, \\
m^{\prime}-d^{\prime} & =m-d, \\
m^{\prime}+d^{\prime} & =(m+d) \cosh \eta-i \sqrt{2} q \sinh \eta .
\end{aligned}
$$

This corresponds to a combination of magnetic gauge and Harrison transformations.

If $\boldsymbol{\alpha}$ and $\boldsymbol{\beta}$ are null, the boosts reduce to rotations and rescaling. Hence the action of the full $S O(1,2)$ component of $H^{\prime}$ is in this case two-parametric. If in some frame

$$
\boldsymbol{\alpha}=\alpha^{0}(1, \mathbf{n}),
$$


where $\mathbf{n}$ is a unit two-dimensional vector, in any other frame one has

$$
\boldsymbol{\alpha}^{\prime}=\alpha^{\prime 0}\left(1, \mathbf{n}^{\prime}\right)
$$

where $\mathbf{n}^{\prime}$ is a rotated version of $\mathbf{n}$. Note that $\boldsymbol{\beta}$ should be transformed using the same parameters. Taking also into account the additional degree of freedom due to the action of $K$, we see that for a given potential $\sigma$ the general type 1 solution depends on three parameters (we do not count here for the asymptotic values of the dilaton and axion fields which can easily be induced once the solutions in the present gauge are given).

Generally both $\boldsymbol{\alpha}^{2}$ and $\boldsymbol{\beta}^{2}$ as well as the scalar product

$$
\boldsymbol{\alpha} \cdot \boldsymbol{\beta}=-2(Q P+A M+N D)
$$

are invariant under the $S O(1,2)$ transformations. These quantities, however, are not preserved by the electric-magnetic duality transformation (4.25), so if one wishes to identify solutions related by symmetries, formally there is no distinction between subcases $a$ and $b$ in the classification of the previous section. However the distinction still may be useful because the corresponding solutions are physically different.

In the general case a rescaling

$$
\boldsymbol{\alpha} \rightarrow k \boldsymbol{\alpha}, \quad \boldsymbol{\beta} \rightarrow k \boldsymbol{\beta},
$$

is also an invariance transformation although trivial, equivalent to a redefinition of harmonic functions, physically it corresponds to an equal rescaling of all six charges, i.e. a change of length scale.

\section{$5 \quad$ Type 1 solutions}

All solutions belonging to the degenerate type 1 can be presented in the form (3.41), generally they depend on two real potentials $\sigma, \tau$ or one complex potential

$$
\zeta=\sigma-i \tau
$$

Contrary to the previous section, here we choose this potential to be dimensionless, so that the $S O(1,2)$ null vector $\boldsymbol{\beta}$ in (3.41) will also be dimensionless. Normalizing this vector so that $\beta^{0}=1$, one can write

$$
\boldsymbol{\beta}=(1, \cos \alpha, \sin \alpha) .
$$

Keeping in mind that from any solution one can generate by $H^{\prime}$ transformations a twoparameter family of solutions, as described in the previous section, we could start from a given value of $\alpha$ (say $\alpha=0$ ) in (5.2). However we find it instructive to leave here the choice of the gauge $\alpha$ open. The corresponding solution (3.41) may be written in complex form

$$
M=A \operatorname{Re}\left\{1+\boldsymbol{\beta} \cdot\left(\boldsymbol{\Gamma}^{2}+i \boldsymbol{\Gamma}^{1}\right) \zeta\right\}
$$

Identifying this with the initial coset representation (3.7) we obtain the sigma-model variables in terms of the harmonic potentials $\sigma$ and $\tau$ :

$$
\begin{aligned}
f^{-1} & =1+\sigma \cos \alpha+\tau \sin \alpha, \\
\chi & =f(\tau \cos \alpha-\sigma \sin \alpha), \\
v+i u & =-f \zeta, \\
z \equiv \kappa+i \mathrm{e}^{-2 \phi} & =i\left(\frac{1+\zeta \cos \alpha}{1+i \zeta \sin \alpha}\right) .
\end{aligned}
$$


Comparing this with the corresponding form of the BPS solutions to the EM system (see Appendix B) one can observe that the scale factor $f^{-1}$ now depends linearly on the harmonic functions, contrasting with a quadratic dependence in the EM case. As we shall see, this has a dramatic effect on the regularity property of the solutions.

Let us consider some particular families of BPS solutions corresponding to different choices of the complex harmonic function $\zeta$.

\section{Extremal rotating Taub-NUT dyon}

We take the harmonic function $\zeta(\boldsymbol{r})$ to be

$$
\zeta=-\frac{\sqrt{2} q}{|\boldsymbol{r}+i \boldsymbol{a}|}
$$

and choose spheroidal coordinates $(r, \theta, \varphi)$ such that 23]

$$
(\boldsymbol{r} \wedge \boldsymbol{a})^{2} \equiv a^{2}\left(r^{2}+a^{2}\right) \sin ^{2} \theta, \quad \boldsymbol{r} \cdot \boldsymbol{a} \equiv \operatorname{arcos} \theta
$$

$(a=|\boldsymbol{a}|)$, and $\varphi$ is the azimuthal angle around $\boldsymbol{a}$. Identifying the physical charges by comparing (4.6) and (5.2), and taking into account the relation

$$
\mathrm{e}^{i \alpha}=-\sqrt{2} \frac{m}{q},
$$

which results from (4.13), we obtain the solution

$$
d s^{2}=\frac{\Delta-a^{2} \sin ^{2} \theta}{\Sigma}(d t+\omega d \varphi)^{2}-\Sigma\left(\frac{d r^{2}}{\Delta}+d \theta^{2}+\frac{\Delta \sin ^{2} \theta}{\Delta-a^{2} \sin ^{2} \theta} d \varphi^{2}\right),
$$

where

$$
\begin{gathered}
\Delta=r^{2}+a^{2}, \\
\Sigma=r^{2}+a^{2} \cos ^{2} \theta+2(N a \cos \theta+M r), \\
\omega=\frac{2\left\{\left(r^{2}+a^{2}\right) N \cos \theta+a M r \sin ^{2} \theta\right\}}{r^{2}+a^{2} \cos ^{2} \theta} .
\end{gathered}
$$

The electric and magnetic potentials are

$$
v=\frac{\sqrt{2}}{\Sigma}(Q r+P a \cos \theta), \quad u=\frac{\sqrt{2}}{\Sigma}(P r-Q a \cos \theta),
$$

and the complex axidilaton is

$$
z=i\left(\frac{r+m-d+i a \cos \theta}{r+m+d+i a \cos \theta}\right) .
$$

The corresponding dilaton factor reads

$$
e^{2 \phi}=\frac{1}{\Sigma}\left|r+2 M+i a \cos \theta-\frac{Q q}{m}\right|^{2} .
$$

This solution corresponds to the BPS limit of the general rotating Taub-NUT dyon solution found in 25] (the radial coordinate used there differs from the present one by a constant shift $r \rightarrow r-2 M)$. For $a \neq 0$ the solution has a naked singularity, as any rotating BPS solution to 
this theory in four dimensions 28]. For $a=0$ the metric has a simple form (previously given by Kallosh et al [10])

$$
d s^{2}=(1+2 M / r)^{-1}(d t+2 N \cos \theta d \varphi)^{2}-(1+2 M / r) d r^{2}-r(r+2 M)\left(d \theta^{2}+\sin ^{2} \theta d \varphi^{2}\right) .
$$

This extreme black hole metric turns out to be singular, the area of the would-be horizon at $r=0$ being zero. It is pointed out in 10 that the monopole string metric $d \bar{s}^{2}=\mathrm{e}^{2 \phi} d s^{2}$ is regular for certain parameter values. Actually we have found that it is always regular, except in the lower-dimensional domain $P M-Q N=0$. The string metric corresponding to (5.13) is

$$
d \bar{s}^{2} \equiv \mathrm{e}^{2 \phi} d s^{2}=\Gamma\left[\frac{(d t+2 N \cos \theta d \varphi)^{2}}{(r+2 M)^{2}}-\frac{d r^{2}}{r^{2}}-d \Omega^{2}\right]
$$

with

$$
\Gamma=(r+\gamma P)^{2}+\gamma^{2} Q^{2}, \quad \gamma=2 \frac{P M-Q N}{Q^{2}+P^{2}} .
$$

For $Q \neq 0, \Gamma$ is positive definite, and the "throat" $r=0$ is at infinite geodesic distance. In the purely magnetic case $Q=0, \Gamma=(r+2 M)^{2}$ leading to the same conclusion, except if $M=0$ (then also $D=0, A=-N, P^{2}=2 N^{2}$ ) where the string metric is massless Taub-NUT. In the special case $P M-Q N=0(\gamma=0)$ with $M \neq 0$, the string metric near $r=0$

$$
d \bar{s}^{2} \sim r^{2}(d t+2 N \cos \theta d \varphi)^{2}-d r^{2}-r^{2} d \Omega^{2}
$$

has a horizon of zero area at the origin $r=0$ of Euclidean space. This "black-point" singularity [29] is mild, all non-radial geodesics being deflected away from $r=0$.

The $a \neq 0$ solution depends on the four parameters $\alpha, q$ (complex), and $a$. The first three parameters correspond to the three degrees of freedom of type 1 solutions under the action of the group $S O(1,2) \times S O(2)$, while the parameter $a$ may be associated with the scale of the dipole potential $\tau$. However we should keep in mind that our monopole-dipole solution has been obtained from the choice (3.40) of the matrices $B, C$ in (3.36). A more general choice solving the constraints $(3.39)$ is, up to $S O(1,2) \times S O(2)$ transformations and rescalings of $\sigma$ and $\tau$,

$$
B=(0, \boldsymbol{\beta}), \quad C=(\boldsymbol{\beta} \cos \psi, \boldsymbol{\beta} \sin \psi) .
$$

The resulting linear form of the matrix $M$

$$
M=A\left\{1+\boldsymbol{\beta} \cdot\left(\boldsymbol{\Gamma}^{1} \tau \cos \psi+\boldsymbol{\Gamma}^{2}(\sigma+\tau \sin \psi)\right)\right\}
$$

with the same potentials $\sigma$ and $\tau$ as in (5.5) leads to the general monopole-dipole BPS solution depending on five parameters (the same solution could alternatively be obtained from (3.41) by transforming the potentials $\sigma \rightarrow \sigma+\tau \sin \psi, \tau \rightarrow \tau \cos \psi)$.

\section{Multicenter solutions}

Let us first discuss the equilibrium conditions between any pair $i, j$ of the multicenter system in which each center in endowed with mass, NUT charge, electric and magnetic charges, and dilaton and axion charges obeying to (4.7), (4.9) and (4.13). The condition that the various attractive (tensor and scalar) and repulsive (vector) forces balance is Eq.(2.24), which translates into

$$
Q_{i} Q_{j}+P_{i} P_{j}=M_{i} M_{j}+N_{i} N_{j}+D_{i} D_{j}+A_{i} A_{j}
$$

or, in terms of complex charges,

$$
\operatorname{Re}\left(q_{i} \bar{q}_{j}-m_{i} \bar{m}_{j}-d_{i} \bar{d}_{j}\right)=0 .
$$


Choosing here the general representation of the matrix $B_{i}$ in terms of three parameters $\mu_{i}, \alpha_{i}$, $\psi_{i}$

$$
\boldsymbol{\alpha}_{i}=2 \mu_{i} \boldsymbol{u}_{i} \cos \psi_{i} \quad \boldsymbol{\beta}_{i}=2 \mu_{i} \boldsymbol{u}_{i} \sin \psi_{i},
$$

where $\boldsymbol{u}_{i}=\left(1, \cos \alpha_{i}, \sin \alpha_{i}\right)$, Eq. (5.19) reads

$$
\boldsymbol{\alpha}_{i} \cdot \boldsymbol{\alpha}_{j}+\boldsymbol{\beta}_{i} \cdot \boldsymbol{\beta}_{j} \equiv 4 \mu_{i} \mu_{j} \cos \left(\psi_{i}-\psi_{j}\right) \boldsymbol{u}_{i} \cdot \boldsymbol{u}_{j}=0 .
$$

This condition has two possible solutions. The first solution is $\boldsymbol{u}_{i} \cdot \boldsymbol{u}_{j}=0$ which, for null vectors $\boldsymbol{u}_{i}, \boldsymbol{u}_{j}$, means $\alpha_{j}=\alpha_{i}$, so that the $\boldsymbol{\alpha}_{j}, \boldsymbol{\beta}_{j}$ are collinear to the $\boldsymbol{\alpha}_{i}, \boldsymbol{\beta}_{i}$; then the whole system of conditions (3.39) is satisfied, so that $B_{i} B_{j}=B_{j} B_{i}=0$, and

$$
M=A\left[1+B_{i} \sigma_{i}+B_{j} \sigma_{j}\right]
$$

is a two-center BPS solution, which can straightforwardly be generalized to any number of centers. The condition (5.22) is also solved by $\cos \left(\psi_{i}-\psi_{j}\right)=0$, or

$$
Q_{i} Q_{j}+P_{i} P_{j}=0
$$

with $\boldsymbol{u}_{i} \cdot \boldsymbol{u}_{j} \neq 0$; the other conditions (3.39) are however not satisfied, so that the equilibrium condition (5.24), which can hold only for a two-center system, does not allow for a geodesic BPS solution.

Now we consider an equilibrium configuration of $n$ centers $\boldsymbol{r}=\boldsymbol{r}_{j}$ endowed with complex masses $m_{j}=M_{j}+i N_{j}$ and charges $q_{j}=Q_{j}+i P_{j}$ (as well as induced axidilaton charges $d_{j}=-q_{j}^{2} / 2 m_{j}$ ) subject to the condition that the angle parameter $\alpha_{j}$ entering the matrix $B_{j}$, or (by virtue of (5.7)) the complex ratio $m_{j} / q_{j}$, is independent of $j$. We also assume that each of these centers carries an arbitrarily oriented dipole moment $\boldsymbol{a}_{j}$. Then, to write down the multi-rotating Taub-NUT dyon solution one merely has to change the complex harmonic function (5.5) to

$$
\zeta=-\sqrt{2} \sum_{j=1}^{n} \frac{q_{j}}{R_{j}}, \quad R_{j}^{2}=\left(\boldsymbol{r}-\boldsymbol{r}_{j}+i \boldsymbol{a}_{j}\right)^{2} .
$$

This solution generalizes the axion-dilaton IWP solution presented by Kallosh et al 10 to the dyon case, presence of NUT's and arbitrary directions of rotation vectors for each center. Clearly, for $\boldsymbol{a}_{j} \neq 0$ we deal with naked singularities.

In the non-rotating case, the metric has the form

$$
d s^{2}=\left(1+\sum_{j=1}^{n} \frac{2 M_{j}}{R_{j}}\right)^{-1}\left(d t+\sum_{j=1}^{n} 2 N_{j} \cos \theta_{j} d \varphi_{j}\right)^{2}-\left(1+\sum_{j=1}^{n} \frac{2 M_{j}}{R_{j}}\right) d \boldsymbol{r}^{2}
$$

where $d \boldsymbol{r}^{2}$ is the flat space line element, and $\theta_{j}, \varphi_{j}$ are polar angles relative to an arbitrarily oriented orthogonal frame centered at $\boldsymbol{r}=\boldsymbol{r}_{\boldsymbol{j}}$. An essential difference with the MajumdarPapapetrou (MP) solution to the EM system (see Appendix B) is that the metric function $f$ now appears as an inverse linear function of Coulomb potentials, while the MP solution (endowed with NUT-s)

$$
d s^{2}=|U|^{-2}(d t+\boldsymbol{\omega} \cdot d \boldsymbol{r})^{2}-|U|^{2} d \boldsymbol{r}^{2},
$$

with

$$
U=1+\sum_{j=1}^{n} \frac{M_{j}+i N_{j}}{R_{j}}, \quad \nabla \wedge \boldsymbol{\omega}=\operatorname{Im}[2 \nabla U+(\bar{U} \nabla U-U \nabla \bar{U})],
$$

has quadratic factors. This is the reason why the surfaces $R_{j}=0$, which for the MP solutions are regular horizons [30], correspond in the dilatonic case to space-time singularities (the radius 
of two-spheres shrinks to zero). It was shown recently [9] that adding a second vector field one can get dilatonic solutions sharing the above MP property too. The other difference between (5.26) and (5.27) is of course the non-linear dependence (5.28), in the EM case, of the rotation one-form $\omega$ on the $N_{i}$ and $M_{i}$ (except if all the $N_{i}$ or all the $M_{i}$ vanish); this is a consequence of Eq.(2.22) for the non-commuting matrices $B$ and $C$ of Appendix b.

\section{Massless states}

BPS solutions with a vanishing Schwarzschild mass are of particular interest in the underlying quantum theory since they can substantially influence the low-energy dynamics [13. As was noticed in the previous section, the type 1 solutions may be massless but at the expense of a non-zero NUT charge (so that the complex mass $m$ remains finite), the BPS condition now reading $Q^{2}+P^{2}=2 N^{2}$. Although they are not asymptotically flat in the usual sense, it is worthwhile giving these solutions in explicit form. All non-rotating massless solutions turn out to possess a locally flat metric

$$
d s^{2}=(d t+2 N \cos \theta d \varphi)^{2}-d r^{2}-r^{2}\left(d \theta^{2}+\sin ^{2} \theta d \varphi^{2}\right),
$$

which is massless Taub-NUT. The dilaton factor is asymmetric with respect to magnetic and electric components:

$$
\mathrm{e}^{2 \phi}=1-\frac{2 Q P}{N r}+\frac{2 Q^{2}}{r^{2}}
$$

so for purely magnetic solutions $\phi \equiv 0$, while for purely electric ones the dilaton field is nonzero, but short-range. In the general dyon case the dilaton charge is non-zero. The axion field

$$
\kappa=\frac{2(A r-N D)}{(r+D)^{2}+(N+A)^{2}}
$$

is non-zero in all cases, the values of the dilaton and axion charges being given by (4.18) or (4.19). In non-dyon cases the axion field is short-range.

Massless multicenter solutions may be constructed in a similar way. In the non-rotating case the metric corresponds to the massless multi-Taub-NUT space-time

$$
d s^{2}=\left(d t+\sum_{j=1}^{n} 2 N_{j} \cos \theta_{j} d \varphi_{j}\right)^{2}-d \boldsymbol{r}^{2} .
$$

Magnetic solutions of this type (with zero dilaton field $\phi$ ) were found earlier [31]. The metric (5.32), as well as the more general metric (5.26), may be asymptotically flat if the sum of the NUT charges vanishes, $\sum_{j} N_{j}=0$.

\section{$6 \quad$ Type 2 solutions}

In the non-degenerate case, the matrix $B$, given by (3.21) where the non-collinear vectors $\boldsymbol{\alpha}$ and $\boldsymbol{\beta}$ are only constrained by (3.26), depends on five parameters. One of these parameters is associated with rescalings of $\sigma$; it is convenient to fix this scale by the condition

$$
\operatorname{det} B=4
$$

(the determinant of $B$, given by eqs. (3.29) and (3.32), is positive by virtue of eq. (3.26)). Then the four non-zero eigenvalues of $B$, solving the characteristic equation $\lambda_{j}^{4}+4=0$, are

$$
\lambda_{j}= \pm(1 \pm i)
$$


The exponential $\mathrm{e}^{B \sigma}$ may be computed using the Lagrange formula

$$
\mathrm{e}^{B \sigma}=\sum_{k=1}^{4} \mathrm{e}^{\lambda_{k} \sigma} \prod_{j \neq k} \frac{B-\lambda_{j}}{\lambda_{k}-\lambda_{j}} .
$$

Substituting (6.3) into (6.4) one obtains

$$
\mathrm{e}^{B \sigma}=g_{0} I+g_{+} B+\frac{1}{2}\left(g_{1} B^{2}+g_{-} B^{3}\right)
$$

where

$$
\begin{gathered}
g_{0}=\cos \sigma \cosh \sigma \\
g_{1}=\sin \sigma \sinh \sigma \\
g_{ \pm}=\frac{1}{2}(\sin \sigma \cosh \sigma \pm \cos \sigma \sinh \sigma) .
\end{gathered}
$$

In the non-degenerate case, BPS solutions to the present system may depend only on one real potential, so the solution (6.4) is likely to be the most general one if $\operatorname{det} B \neq 0$. Using Eqs. (3.21), (3.25) and (3.27) one obtains the exponential as a linear combination of matrices $\Gamma^{a}=\left(\Gamma_{0}^{a}, \Gamma_{1}^{a}, \Gamma_{1}^{a}\right)$ forming a triplet of $1+2$ Clifford algebras:

$$
\mathrm{e}^{B \sigma}=g_{0} I+\boldsymbol{v}_{a} \cdot \boldsymbol{\Gamma}^{a}
$$

where

$$
\begin{aligned}
& \boldsymbol{v}_{0}=-g_{1} \boldsymbol{\alpha} \wedge \boldsymbol{\beta}, \\
& \boldsymbol{v}_{1}=g_{+} \boldsymbol{\alpha}-g_{-} \boldsymbol{\alpha}^{\prime}, \\
& \boldsymbol{v}_{2}=g_{+} \boldsymbol{\beta}-g_{-} \boldsymbol{\beta}^{\prime} .
\end{aligned}
$$

Comparing the two representations (3.7) and (3.9) of $M \in S p(4, R) / U(2)$ we obtain the following expressions for the potentials in terms of the components $v_{a}^{b}$ of the vectors $\boldsymbol{v}_{a}$ :

$$
\begin{aligned}
f^{-1} & =g_{0}+v_{0}^{0}+v_{1}^{2}+v_{2}{ }^{1}, \\
\chi f^{-1} & =v_{1}{ }^{1}-v_{2}^{2}, \\
-v f^{-1} & =v_{0}{ }^{1}+v_{2}^{0} \\
u f^{-1} & =v_{0}^{2}+v_{1}^{0} \\
\mathrm{e}^{2 \phi}-v^{2} f^{-1} & =g_{0}-v_{0}^{0}+v_{1}^{2}-v_{2}{ }^{1} \\
\kappa \mathrm{e}^{2 \phi}-u v f^{-1} & =v_{1}{ }^{1}+v_{2}^{2} .
\end{aligned}
$$

Substituting Eqs. (6.6) and (6.8) one finds for the metric function $f$ the following expression

$$
f^{-1}=\cos \sigma\left(\cosh \sigma+\mu_{+} \sinh \sigma\right)+\sin \sigma\left(\mu_{0} \sinh \sigma+\mu_{-} \cosh \sigma\right),
$$

where

$$
\mu_{0}=\Lambda^{-2}\left(|m|^{2}-|d|^{2}\right), \quad \mu_{ \pm}=\Lambda^{-1} M \pm \Lambda^{-3} \operatorname{Re}\left[\bar{q}\left(q^{2}+2 m d\right)\right]
$$

with the normalization factor $\Lambda=|\boldsymbol{\alpha} \wedge \boldsymbol{\beta}|^{1 / 2}$ for the $\boldsymbol{\alpha}, \boldsymbol{\beta}$ of (4.6). We then obtain for the electric and magnetic potentials,

$$
v+i u=\sqrt{2} f\left\{q g_{+}+(q \bar{m}+\bar{q} d) \Lambda^{-1} g_{1}+\bar{q}\left(q^{2}+2 m d\right) \Lambda^{-2} g_{-}\right\} .
$$

The corresponding dilaton factor is

$$
\mathrm{e}^{2 \phi}=v^{2} f^{-1}+\cos \sigma\left(\cosh \sigma+\nu_{+} \sinh \sigma\right)-\sin \sigma\left(\mu_{0} \sinh \sigma-\nu_{-} \cosh \sigma\right),
$$


where

$$
\nu_{ \pm}=D \pm \Lambda^{-2} \operatorname{Re}\left[\bar{m}\left(q^{2}+2 m d\right)\right] .
$$

Points where $f^{-1}=0$, i.e.

$$
\tan \sigma=-\frac{\cosh \sigma+\mu_{+} \sinh \sigma}{\mu_{-} \cosh \sigma+\mu_{0} \sinh \sigma}
$$

correspond to spacetime singularities?. Since $f^{-1}$ enters into the spacetime metric (2.2) as a conformal factor of the three-space, these singularities (which are also singularities of the electric and magnetic potentials (6.11) are point singularities. For a one-center solution $\sigma=$ $k / r$, the range of $r$ is therefore $] r_{1},+\infty$ [, where $r_{1}>0$ is the largest value of $r$ solving eq. (6.14). The behaviour of the static $(\chi=0)$ metric near $r=r_{1}$ is

$$
d s^{2} \sim c^{-2} \rho^{-2 / 3} d t^{2}-d \rho^{2}-r_{1}^{2} c^{2} \rho^{2 / 3} d \Omega^{2}
$$

with $\rho^{2 / 3} \propto\left(r-r_{1}\right)$. One can show that all non-radial geodesics, as well as radial timelike geodesics, are deflected away from $\rho=0\left(r=r_{1}\right)$. Only radial, lightlike geodesics terminate at the singularity $\rho=0$, so this is a very weak singularity. Again, the corresponding string metric $d \bar{s}^{2}=\mathrm{e}^{2 \phi} d s^{2}$ turns out to be regular. Generically the dilaton factor $\mathrm{e}^{2 \phi}$ has the same poles as $f$ so that, near $r=r_{1}$,

$$
d \bar{s}^{2} \sim \frac{a^{2}}{\left(r-r_{1}\right)^{2}} d t^{2}-d r^{2}-r_{1}^{2} d \Omega^{2} .
$$

All timelike or lightlike geodesics are deflected away from the throat $r=r_{1}$, except for radial lightlike geodesics which reach the throat after an infinite "affine time".

The same local solution (6.8) may also be extended to the ranges $r \in] r_{k+1}, r_{k}[$, where $\sigma_{k} \equiv \sigma\left(r_{k}\right)$ and $\sigma_{k+1}$ are two consecutive roots of (6.14); the corresponding spatial sections are compact, with two point singularities $r=r_{k}$ and $r=r_{k+1}$. The more general $n$-center harmonic potential $\sigma=\sum_{j=1}^{n} k_{j} /\left|\boldsymbol{r}-\boldsymbol{r}_{\boldsymbol{j}}\right|$ leads to an asymptotically flat/Taub-NUT solution with $p$ point singularities, where $p$ is the number of connected components of the "surface" $\sigma(\boldsymbol{r})=\sigma_{1}$, as well as compact solutions with point singularities for $\sigma_{k}<\sigma(\boldsymbol{r})<\sigma_{k+1}$. For the corresponding string metrics, the point singularities are replaced by throats at infinite geodesic distance.

These non-degenerate solutions may have scalar charges independent of the electromagnetic ones (up to the BPS condition). Let us consider some particular cases starting with type 2b $(\boldsymbol{\alpha}, \boldsymbol{\beta}$ non-null) which leads to surprisingly simple formulas. Here one discovers counterparts of the type 1 extreme Reissner-Nordström black holes in the dilaton-axion theory which do not possess dilaton charges.

1. Electrically charged "Reissner-Nordström":

$$
f=\mathrm{e}^{2(\phi-\sigma)}=\frac{\mathrm{e}^{-\sigma}}{\sqrt{2} \cos (\sigma-\pi / 4)}, \quad v=\frac{\sin \sigma}{\cos (\sigma-\pi / 4)}, \quad \sigma=M / r .
$$

It has $Q=M$, all the other charges being zero. The point singularity of the Einstein metric is located at $r_{1}=4 M / 3 \pi$, which is also a singularity of the electric and dilaton fields. Note that the non-zero dilaton field is short range. The string metric is, as discussed above, regular with a throat $r=4 M / 3 \pi$ at infinite geodesic distance. In contrast, for the type 1 extreme Reissner-Nordström solution with $-D=M=Q / \sqrt{2}$, both the Einstein and string metrics are singular (the coefficient $\gamma$ in (5.15) vanishes).

\footnotetext{
${ }^{3}$ In the case of the corresponding KK solutions of type 1 [22], the function $f^{-1}$ similarly vanishes for a denumerable set of values of $\sigma$. These four-dimensional spacetime singularities are due to a breakdown of the Kaluza-Klein dimensional reduction, the five-dimensional metric being everywhere regular.
} 
2. Magnetically charged "Reissner-Nordström":

$$
f=\mathrm{e}^{-2(\phi+\sigma)}=\frac{\mathrm{e}^{-\sigma}}{\sqrt{2} \cos (\sigma-\pi / 4)}, \quad u=\frac{\sin \sigma}{\cos (\sigma-\pi / 4)}, \quad \sigma=M / r
$$

which corresponds to $P=M$ and has a similar singularity structure. However in this case the product $f \mathrm{e}^{2 \phi}=\mathrm{e}^{-2 \sigma}$ is non-singular, which results in the string metric $d \bar{s}^{2}$ being singular (radial geodesics terminate at the point singularity $r=4 M / 3 \pi$ ).

These solutions may be subjected to $S O(1,2) \times S O(2)$ transformations to get non-zero values of other charges. In particular, one can obtain massless asymptotically flat solutions (without NUT's).

3. Massless electrically charged "geon" possessing a dilaton charge:

$$
f=\mathrm{e}^{2(\phi-\sigma)}=\frac{\mathrm{e}^{\sigma}}{\sqrt{2} \cos (\sigma-\pi / 4)}, \quad v=\frac{\sin \sigma}{\cos (\sigma-\pi / 4)}, \quad \sigma=Q / r .
$$

It has $D=-Q, M=N=A=P=0$. It is worth noting that the gravitational field is non-zero, but decays as $r^{-2}$.

All the above solutions are static.

4. Massless magnetically charged "geon" with axion charge $A=P$ :

$$
\begin{aligned}
& f^{-1}=\cos \sigma \cosh \sigma-\sin \sigma \sinh \sigma, \quad \chi=f(\sin \sigma \cosh \sigma-\cos \sigma \sinh \sigma) \\
& v=u \tanh \sigma=\sqrt{2} f \sin \sigma \sinh \sigma, \quad \mathrm{e}^{2 \phi}=f\left(\cos ^{2} \sigma+\sinh ^{2} \sigma\right) \\
& \kappa=f \mathrm{e}^{-2 \phi}(\sin \sigma \cosh \sigma+\cos \sigma \sinh \sigma)
\end{aligned}
$$

where $\sigma=P / r$. This solution possesses short-range electric and dilaton fields. The singularity corresponds to

$$
\cot \sigma=\tanh \sigma
$$

Note that for solutions of type $2 \mathrm{~b}$ the only restriction on the charges is the BPS condition (4.7). Now consider type 2a solutions (both $\boldsymbol{\alpha}, \boldsymbol{\beta}$ are null). In this case there is a second constraint on the charges, so that the dilaton and axion charges are functions of the other charges (this dependence is given by the relations (4.22), (4.23) different from those for dilatonaxion extreme black holes, (4.12)).

5. Massive symmetric Reissner-Nordström $Q=-P=M / \sqrt{2}$ without dilaton and axion charges:

$$
\begin{aligned}
& f=\frac{\mathrm{e}^{-\sigma}}{\sqrt{2} \cos (\sigma-\pi / 4)}, \quad u=-v=\frac{\sin \sigma}{\cos (\sigma-\pi / 4)}, \\
& \mathrm{e}^{2 \phi}=\frac{\cosh \sigma+\mathrm{e}^{-\sigma} \sin \sigma \cos \sigma}{\sqrt{2} \cos (\sigma-\pi / 4)}, \quad \kappa=\frac{\mathrm{e}^{-\sigma} \sin \sigma \cos \sigma-\sinh \sigma}{\cosh \sigma+\mathrm{e}^{-\sigma} \sin \sigma \cos \sigma}, \quad \sigma=M / r .
\end{aligned}
$$

Note that, as in the non-dyon cases, although the dilaton and axion charges are zero, there still exist short-range dilaton and axion fields.

6. Massless symmetric dyon $P=-Q=A / \sqrt{2}$ without dilaton charge:

$$
\begin{aligned}
& f=\frac{\mathrm{e}^{\sigma}}{\sqrt{2} \cos (\sigma-\pi / 4)}, \quad u=-v=\frac{\sin \sigma}{\cos (\sigma-\pi / 4)}, \\
& \mathrm{e}^{2 \phi}=\frac{\cosh \sigma+\mathrm{e}^{\sigma} \sin \sigma \cos \sigma}{\sqrt{2} \cos (\sigma-\pi / 4)}, \quad \kappa=\frac{\sinh \sigma+\mathrm{e}^{\sigma} \sin \sigma \cos \sigma}{\cosh \sigma+\mathrm{e}^{\sigma} \sin \sigma \cos \sigma}, \quad \sigma=A / r .
\end{aligned}
$$

This solution corresponds to an interchange $\boldsymbol{\alpha} \leftrightarrow \boldsymbol{\beta}$ in the previous solution. 


\section{Discussion}

We have presented a constructive approach to the generation of BPS solutions to gravitycoupled field theories admitting a $\sigma$-model representation in three dimensions. This purely bosonic approach has the advantage of reducing all calculations to algebraic manipulations with matrices representing relevant cosets (no differential equations have to be solved). It yields solutions in a closed form readily parametrized by physical charges and automatically symmetric under the dualities preserving asymptotic flatness. In addition, it provides an algebraic classification of solutions according to the possible algebraic types of generating matrices.

For the particular case of dilaton-axion gravity with one vector field (which was extensively explored during recent years) we have listed all stationary asymptotically flat BPS solutions. These fall into two classes. The first class includes the well-known multi-extreme black holes and their rather obvious generalizations. The corresponding Einstein metrics have singular event horizons (null point singularities). In the case of the solutions of the new, second class, dilaton and axion charges are not tightly bound to electric/magnetic charges. Their Einstein metrics have mild timelike naked singularities. However the associated string metrics are generically regular for both classes of solutions.

Both of these classes contain massless states. In the degenerate case (first class), the vanishing of mass can be achieved at the expense of the introduction of NUT's (although for multicenter configurations the total NUT charge may still be zero). In the non-degenerate case (new class), there are strictly asymptoticaly flat massless solutions.

After this work was completed, we received a preprint by Kechkin and Yurova (hepth/9604071] in which the results of [20] and 22] are also used to construct classes of stationary BPS solutions to dilaton-axion gravity. However these authors incorrectly identify the coset to which the matrices $\mathrm{e}^{B \sigma}$ belong as $S p(4, R) / G L(2, R)$. As we have seen, the correct identification is $S p(4, R) /(S O(1,2) \times S O(2))$, the $\mathrm{SO}(2)$ transformations corresponding to electric-magnetic duality rotations. Neither the BPS solutions depending on two harmonic potentials nor our type 2 solutions are considered in this paper.

Acknowledgements D.G. wishes to thank the Laboratoire de Gravitation et Cosmologie Relativistes (UPMC/CNRS) for hospitality during his visit while this work was initiated. He thanks R. Kerner for useful discussions, and R. Kallosh for stimulating correspondence. He is also grateful to FAPESP for financial support and to P. Letelier for his kind invitation to visit Campinas University while the final version of this paper was completed. This work was supported in part by the RFBR grant $96-02-18899$.

\section{APPENDIX A: $S p(4, R)$ and 1+2 Clifford algebras}

The Lie algebra of $S p(4, R)$ consists of ten real $4 \times 4$ matrices $Y$ satisfying the condition

$$
Y^{T} J+J Y=0
$$

where $J$ is given by (3.13). For further analysis it is convenient to introduce the full set of sixteen independent real $4 \times 4$ matrices as tensor products of two sets of $2 \times 2$ matrices

$$
e_{\mu \nu}=\rho_{\mu} \otimes \sigma_{\nu}, \quad(\mu, \nu=0,1,2,3),
$$

with

$$
\rho_{0}=\sigma_{0}=I, \rho_{1}=\sigma_{1}=\sigma_{x}, \rho_{2}=\sigma_{2}=i \sigma_{y}, \rho_{3}=\sigma_{3}=\sigma_{z}
$$


where $\sigma_{x}, \sigma_{y}, \sigma_{z}$ are the standard Pauli matrices, and it is assumed that $\rho_{\mu}$ act on $2 \times 2$ blocks, while $\sigma_{\mu}$ act inside these blocks. The following multiplication rules hold

$$
\sigma_{2} \sigma_{1}=\sigma_{3}, \quad \sigma_{3} \sigma_{2}=\sigma_{1}, \quad \sigma_{3} \sigma_{1}=\sigma_{2}, \quad \sigma_{1}^{2}=-\sigma_{2}^{2}=\sigma_{3}^{2}=I .
$$

In this notation, the matrices introduced in Sect. 3 read $A=e_{03}, K=e_{23}$, while the full Lie algebra $s p(4, R)$ satisfying $(\mathrm{A} .1)$ is

$$
s p(4, R)=\left\{e_{02}, e_{10}, e_{11}, e_{13}, e_{20}, e_{21}, e_{23}, e_{30}, e_{31}, e_{33}\right\}
$$

We look for a parametrization of the coset $S p(4, R) / U(2)$ (choosing as representatives the symmetric symplectic matrices $M$ ) of the type

$$
M=A \mathrm{e}^{B \sigma}, \quad A=e_{03}, \quad A^{2}=I,
$$

or,

$$
\mathrm{e}^{B \sigma}=A M
$$

Since $A \in S p(4, R) / U(2)$, the matrix $B$ belongs to $s p(4, R)$,

$$
J B J=B^{T}
$$

and is subject to the symmetry condition

$$
A B A=B^{T}
$$

The exponential $\mathrm{e}^{B \sigma}$ is therefore a non-symmetric matrix which can be shown to belong to the coset space $S p(4, R) /(S O(2) \times S O(1,2))$. Indeed, solving the equations (A.6) and (A.5) one finds that $B \in \mathcal{B}$, with

$$
\mathcal{B}=\left\{e_{02}, e_{10}, e_{13}, e_{21}, e_{30}, e_{33}\right\} .
$$

The remaining generators of $S p(4, R)$ form a subalgebra

$$
\mathcal{H}^{\prime}=\left\{e_{11}, e_{20}, e_{23}, e_{31}\right\}
$$

which can be identified with $s o(2) \oplus s o(1,2)$, where the $s o(2)$ component is $K=e_{23}$, while the so $(1,2)$ consists of

$$
\Sigma_{a}=\left\{e_{20},-e_{11}, e_{31}\right\}
$$

$(a=0,1,2$ is an $s o(1,2)$ index $)$ with $\Sigma_{0} \equiv J$, so that

$$
\left[\Sigma_{a}, \Sigma_{b}\right]=2 \varepsilon_{a b}^{c} \Sigma_{c}, \quad\left[K, \Sigma_{a}\right]=0
$$

(the three-dimensional Levi-Civita symbol is defined by $\varepsilon_{012}=1$, and the indices are raised and lowered with the $1+2$ metric $\eta_{a b}=\eta^{a b}=\operatorname{diag}(1,-1,-1)$ ). All the elements of $\mathcal{H}^{\prime}$ satisfy

$$
A \mathcal{H}^{\prime} A=-\mathcal{H}^{\prime T}
$$

which ensures that the similarity transformations of $B$ generated by $\mathcal{H}^{\prime}$ preserve the symmetry condition (A.6).

Inside $\mathcal{B}$ one can identify two sets of $1+2$ Clifford algebras:

$$
\begin{aligned}
& \Gamma_{a}^{1}=\left\{e_{21},-e_{10}, e_{30}\right\} \\
& \Gamma_{a}^{2}=\left\{e_{02}, e_{33}, e_{13}\right\}
\end{aligned}
$$


satisfying the anticommutation relations

$$
\left\{\Gamma_{a}^{1}, \Gamma_{b}^{1}\right\}=\left\{\Gamma_{a}^{2}, \Gamma_{b}^{2}\right\}=-2 \eta_{a b} I .
$$

Their relation to the $s o(2)$ subalgebra is clear from

$$
\begin{aligned}
& {\left[K, \Gamma_{a}^{1}\right]=-2 \Gamma_{a}^{2},} \\
& {\left[K, \Gamma_{a}^{2}\right]=2 \Gamma_{a}^{1},} \\
& {\left[\Gamma_{a}^{1}, \Gamma_{b}^{2}\right]=2 K \eta_{a b},}
\end{aligned}
$$

that is, $K$ mixes the two sets. Contrary to this, the $s o(1,2)$ does not mix them:

$$
\begin{aligned}
& {\left[\Sigma_{a}, \Gamma_{b}^{1}\right]=2 \varepsilon_{a b}{ }^{c} \Gamma_{c}^{1},} \\
& {\left[\Sigma_{a}, \Gamma_{b}^{2}\right]=2 \varepsilon_{a b}{ }^{c} \Gamma_{c}^{2},} \\
& {\left[\Gamma_{a}^{1}, \Gamma_{b}^{1}\right]=\left[\Gamma_{a}^{2}, \Gamma_{b}^{2}\right]=2 \varepsilon_{a b}{ }^{c} \Sigma_{c} .}
\end{aligned}
$$

Remarkably, the anticommutator of $\boldsymbol{\Gamma}^{1}$ and $\boldsymbol{\Gamma}^{2}$ generates a third $1+2$ Clifford algebra, $\boldsymbol{\Gamma}^{0}$ :

$$
\left\{\Gamma_{a}^{1}, \Gamma_{b}^{2}\right\}=-2 \varepsilon_{a b}{ }^{c} \Gamma_{c}^{0}, \quad \Gamma_{a}^{0}=\left\{e_{03}, e_{32}, e_{12}\right\}
$$

consisting of matrices which do not belong to $s p(4, R)$, and satisfying

$$
\left\{\Gamma_{a}^{0}, \Gamma_{b}^{0}\right\}=2 \eta_{a b} I .
$$

This new set commutes with the $s o(2) \in \mathcal{H}^{\prime}$

$$
\left[K, \Gamma_{a}^{0}\right]=0
$$

while for commutators with the $s o(1,2) \in \mathcal{H}^{\prime}$ one has, similarly to (A.13),

$$
\begin{aligned}
& {\left[\Sigma_{a}, \Gamma_{b}^{0}\right]=2 \varepsilon_{a b}{ }^{c} \Gamma_{c}^{0},} \\
& {\left[\Gamma_{a}^{0}, \Gamma_{b}^{0}\right]=-2 \varepsilon_{a b}{ }^{c} \Sigma_{c} .}
\end{aligned}
$$

Comparing Eqs. (A.11), (A.13), (A.14), (A.17) one can see that the two-index $S O(1,2)$ covariant relations (3.24) hold indeed. Now, taking the commutator

$$
\left[\Gamma_{a}^{c}, \Gamma_{b}^{d}\right]=-2 \eta^{c d} \varepsilon_{a b}{ }^{e} \Sigma_{e}-2 \varepsilon^{c d}{ }_{e} \eta_{a b} K^{e}
$$

one finds that the previously introduced $K$ is just the "time" component $K=K^{0}$ of the "vector"

$$
K^{a}=\left\{e_{23},-e_{01}, e_{22}\right\}
$$

which generates the contravariant $S O(1,2)$ algebra dual to the "covariant" $S O(1,2)$ algebra generated by the $\Sigma_{a}$ :

$$
\begin{aligned}
& {\left[K^{a}, K^{b}\right]=2 \varepsilon^{a b}{ }_{c} K^{c},} \\
& {\left[K^{a}, \Gamma_{c}^{b}\right]=2 \varepsilon^{a b}{ }_{e} \Gamma^{e}{ }_{c},} \\
& {\left[K^{a}, \Sigma_{b}\right]=0 .}
\end{aligned}
$$


The previously introduced tensor set $\Gamma_{b}^{a}$ can be expressed as the tensor product of the two vector sets

$$
K^{a} \Sigma_{b}=\Gamma_{b}^{a} .
$$

Together with the unit matrix, the set $\left\{K^{a}, \Sigma_{b}, \Gamma_{b}^{a}\right\}$ constitutes a complete basis of the space of real $4 \times 4$ matrices.

\section{APPENDIX B: Sigma model construction of the Israel-Wilson- Perjès solutions}

We show here how the well-known Israel-Wilson-Perjès [23] solutions of the EinsteinMaxwell field equations may be recovered from the general sigma model construction outlined in Sect. 2.

We first introduce the familiar Ernst potentials 15

$$
\mathcal{E}=f+i \chi-\bar{\Phi} \Phi, \quad \Phi=\frac{1}{\sqrt{2}}(v+i u)
$$

in terms of which the target space metric (3.6) reduces, for vanishing dilaton and axion fields, $\phi=\kappa=0$, to

$$
d l^{2}=\frac{1}{2 f^{2}}|d \mathcal{E}+2 \bar{\Phi} d \Phi|^{2}-\frac{2}{f} d \Phi d \bar{\Phi} .
$$

This may be identified as the metric of the symmetric space $\mathrm{SU}(2,1) / \mathrm{S}(\mathrm{U}(2) \times \mathrm{U}(1))$. A matrix representative of this coset may be chosen as 18

$$
M=f^{-1}\left(\begin{array}{ccc}
1 & \sqrt{2} \Phi & i(\overline{\mathcal{E}}-\mathcal{E}+2 \Phi \bar{\Phi}) / 2 \\
\sqrt{2} \bar{\Phi} & -(\mathcal{E}+\overline{\mathcal{E}}-2 \Phi \bar{\Phi}) / 2 & -i \sqrt{2} \mathcal{E} \bar{\Phi} \\
i(\overline{\mathcal{E}}-\mathcal{E}-2 \Phi \Phi) / 2 & i \sqrt{2} \overline{\mathcal{E}} \Phi & \mathcal{E} \overline{\mathcal{E}}
\end{array}\right)
$$

This matrix is hermitean, $M^{+}=M$, and belongs to $\mathrm{SU}(2,1), M^{+} J M=J$, $\operatorname{det} M=1$, with

$$
J=\left(\begin{array}{ccc}
0 & 0 & -i \\
0 & 1 & 0 \\
i & 0 & 0
\end{array}\right)
$$

An asymptotically flat (or Taub-NUT) solution of the EM field equations depending on one harmonic potential $\sigma$ may be written in the exponential form (2.12), where the constant matrix

$$
A=\left(\begin{array}{ccc}
1 & 0 & 0 \\
0 & -1 & 0 \\
0 & 0 & 1
\end{array}\right)
$$

commutes with $J$. The matrix $B$ belongs to $\operatorname{su}(2,1)$,

$$
B^{+} J+J B=0, \quad \operatorname{Tr} B=0,
$$

and is further constrained by the symmetry condition

$$
B^{+} A-A B=0 \text {. }
$$

These conditions also imply $\operatorname{det} B=0$. The characteristic identity (3.32) for the matrix $B$ then reduces to

$$
B^{3}=\frac{1}{2} \operatorname{Tr}\left(B^{2}\right) B,
$$


so that the exponential representation (2.12) may be rewritten as

$$
M=A\left[1+t^{-1} B \sinh t \sigma+t^{-2} B^{2}(\cosh t \sigma-1)\right],
$$

where $t^{2} \equiv(1 / 2) \operatorname{Tr}\left(B^{2}\right)$.

Solutions corresponding to null geodesics in target space satisfy the further condition

$$
\operatorname{Tr}\left(B^{2}\right)=0 .
$$

The matrices B solving conditions (B.6), (B.7) and (B.10) belong to a single matrix type, with $B^{3}=0, B^{2} \neq 0$, depending (up to an overall multiplicative constant) on two real parameters $\alpha, \beta$ :

$$
B=\left(\begin{array}{ccc}
2 \cos \alpha & \sqrt{2} \mathrm{e}^{-i \beta} & -2 \sin \alpha \\
-\sqrt{2} \mathrm{e}^{i \beta} & 0 & i \sqrt{2} \mathrm{e}^{i \beta} \\
-2 \sin \alpha & i \sqrt{2} \mathrm{e}^{-i \beta} & -2 \cos \alpha
\end{array}\right) .
$$

Two such matrices $B$ and $B^{\prime}$ are orthogonal if

$$
\operatorname{Tr}\left(B B^{\prime}\right) \equiv 8\left[\cos \left(\alpha-\alpha^{\prime}\right)-\cos \left(\beta-\beta^{\prime}\right)\right]=0 .
$$

The commutator $\left[B, B^{\prime}\right]$ does not vanish (unless $B^{\prime}= \pm B$ ), but commutes with $B$ and $B^{\prime}$ iff

$$
\alpha^{\prime}+\beta^{\prime}=\alpha+\beta,
$$

which also solves (B.12). So the matrices $B_{a}$ belonging to the class $(\mathrm{B} .13)$ may be used to construct a solution (2.19) depending on several harmonic functions $\sigma_{a}$. However only two of these matrices are linearly independent. One can choose a basis consisting of $B$ and of the matrix $C$ defined by $\alpha^{\prime}=\alpha-\pi / 2, \beta^{\prime}=\beta+\pi / 2$, e.g.

$$
C=\left(\begin{array}{ccc}
2 \sin \alpha & -i \sqrt{2} \mathrm{e}^{-i \beta} & 2 \cos \alpha \\
-i \sqrt{2} \mathrm{e}^{i \beta} & 0 & -\sqrt{2} \mathrm{e}^{i \beta} \\
2 \cos \alpha & \sqrt{2} \mathrm{e}^{-i \beta} & -2 \sin \alpha
\end{array}\right) .
$$

This choice, such that

$$
B C+C B=0, \quad C^{2}=B^{2},
$$

leads to the simple form for the solution depending on two potentials

$$
M=A \mathrm{e}^{B \sigma+C \tau}=A\left[1+B \sigma+C \tau+\frac{1}{2} B^{2}\left(\sigma^{2}+\tau^{2}\right)\right] .
$$

Combining the two real potentials $\sigma, \tau$ into a single complex harmonic potential

$$
\zeta=\sigma-i \tau
$$

we recover from $(\overline{\mathrm{B} .16})$ and $(\overline{\mathrm{B} .3})$ the Ernst potentials

$$
\mathcal{E}=\frac{1-\mathrm{e}^{i \alpha} \zeta}{1+\mathrm{e}^{i \alpha} \zeta}, \quad \Phi=\frac{\mathrm{e}^{-i \beta} \zeta}{1+\mathrm{e}^{i \alpha \zeta}},
$$

leading to the gravitational potentials

$$
\begin{aligned}
f^{-1} & =\left|1+\mathrm{e}^{i \alpha} \zeta\right|^{2}=1+2(\sigma \cos \alpha+\tau \sin \alpha)+\sigma^{2}+\tau^{2}, \\
\chi & =2 f(\tau \cos \alpha-\sigma \sin \alpha),
\end{aligned}
$$

to be compared with the solution (5.4) for dilaton-axion gravity. Eq. (B.18) implies the linear relation between the Ernst potentials

$$
\Phi=\frac{1}{2} \mathrm{e}^{-i(\alpha+\beta)}(1-\mathcal{E}),
$$

which is equation $(24)$ of Israel and Wilson [23], while their harmonic potential $2 /(1+\mathcal{E})-1$ is our $\mathrm{e}^{i \alpha} \zeta$. 


\section{References}

[1] E.B. Bogomol'nyi, Yad. Fiz. 24, 861 (1976) (Sov. J. Nucl. Phys. 4, 449 (1976)).

[2] E. Witten and D. Olive, Phys. Lett. B78, 97 (1978).

[3] E. Witten, Comm. Math. Phys. 80, 381 (1981); J.M. Nester, Phys. Lett. A 83, 241 (1981); W. Israel and J.M. Nester, Phys. Lett. A 83, 241 (1981); C.M. Hull, Comm. Math. Phys. 90, 545 (1983).

[4] G.W. Gibbons, Soliton States and Central Charges in Extended Supergravity Theories, in Unified Theories of Elementary Particles, Lecture Notes in Physics, Vol. 160, edited by P. Breitenlohner and H.P. Dürr, Springer Verlag, 1982.

[5] G.W. Gibbons and C.M. Hull, Phys. Lett. B 109, 190 (1982); C.M. Hull, Comm. Math. Phys. 90, 545 (1983).

[6] R. Kallosh, Phys. Lett. B 282, 80 (1992).

[7] G.W. Gibbons, Nucl. Phys. B 207, 337 (1982).

[8] R. Kallosh, A. Linde, T. Ortín, A. Peet, and A. van Proyen, Phys. Rev. D 46, 5278 (1992); R. Kallosh, T. Ortín, Phys. Rev. D 48, 742 (1993); R. Kallosh, Phys. Rev. D 52, 1234 (1995).

[9] E. Bergshoeff, R. Kallosh, and T. Ortín, Stationary Axion/Dilaton Solutions and Supersymmetry, preprint UG-3/96 (hep-th/9605059).

[10] R. Kallosh, D. Kastor, T. Ortín, and T. Torma, Phys. Rev. D 50, 6374 (1994).

[11] J. Shwarz and A. Sen, Phys. Lett. B 312, 105 (1993); A. Sen, Mod. Phys. Lett, A 8, 2023 (1993); M.J. Duff, J.T. Liu, and J. Rahmfeld, Nucl. Phys. B 459, 125 (1996).

[12] M. Cvetič and D. Youm, Phys. Lett. B 359, 87 (1995); M. Cvetič and D. Youm, Phys. Rev. D 53, R584 (1996); K. Behrndt and R. Kallosh Phys. Rev. D 53, R589 (1996); M. Cvetič and A. Tseytlin, Phys. Rev. D 53, 5619 (1996); M. Cvetič and A. Tseytlin, Non-Extreme Black Holes from Non-Extreme Intersecting M-branes, preprint DAMTP/R/96/27 (hep-th/9606033); M. Cvetič and C.M. Hull, Black Holes and UDuality, preprint DAMTP/R/96/31, (hep-th/9606193).

[13] A. Strominger, Nuclear Physics 45196 (1995); C. Hull and P. Townsend, Nuclear Physics 451525 (1995).

[14] K.P. Tod, Phys. Lett. B 121, 241 (1983); Class. Quant. Grav. 12, 1801 (1995).

[15] F.J. Ernst, Phys. Rev. 167, 1175 (1968); 168, 1415 (1968); W. Kinnersley, Journ. Math. Phys. 14, 651 (1973); 18, 1529 (1977).

[16] D. Maison, Gen. Rel. and Grav. 10, 717 (1979).

[17] G. Neugebauer and D. Kramer, Ann. der Physik (Leipzig) 24, 62 (1969).

[18] P.O. Mazur, Acta Phys. Polon. B14, 219 (1983); A. Eris, M. Gürses, and A. Karasu, Journ. Math. Phys. 25, 1489 (1984).

[19] P. Breitenlohner, D. Maison, and G. Gibbons, Comm. Math. Phys. 120, 253 (1988). 
[20] D.V. Gal'tsov, Phys. Rev. Lett. 74, 2863 (1995).

[21] D. Kramer, H. Stephani, M. MacCallum, and E. Herlt, Exact Solutions of the Einstein Field Equations, CUP, 1980.

[22] G. Clément, Gen. Rel. and Grav. 18, 861 (1986); Phys. Lett. A 118, 11 (1986).

[23] W. Israel and G.A. Wilson, Journ. Math. Phys. 13865 (1972); Z. Perjès, Phys. Rev. Lett. 271668 (1971).

[24] R.D. Sorkin, Phys. Rev. Lett. 5187 (1983); D.J. Gross and M.J. Perry, Nucl. Phys. B 22629 (1983).

[25] D.V. Gal'tsov and O.V. Kechkin, Phys. Rev. D50 7394 (1994).

[26] B. Julia, in Unified field theories in more than 4 dimensions, V. De Sabbata, E. Schmutzer (eds.), Singapore, WS 1983.

[27] D.V. Gal'tsov and O.V. Kechkin, Phys. Lett. B361 52 (1995).

[28] H. Horowitz and A. Sen, Phys. Rev. D 53808 (1996).

[29] G.W. Gibbons and K. Maeda, Nucl. Phys. B 298741 (1988); D. Garfinkle, G.T. Horowitz and A. Strominger, Phys. Rev. D 433140 (1991); H.H. Soleng, Phys. Rev. D 526178 (1995).

[30] J.B. Hartle and S.W. Hawking, Comm. Math. Phys. 2687 (1972).

[31] D.V. Gal'tsov and O.V. Kechkin, Phys. Rev. D 541656 (1996). 